\title{
Role of cAMP Cascade in Synaptic Stability and Plasticity: Ultrastructural and Physiological Analyses of Individual Synaptic Boutons in Drosophila Memory Mutants
}

\author{
John J. Renger, ${ }^{1}$ Atsushi Ueda, ${ }^{1}$ Harold L. Atwood,, ${ }^{1,2}$ C. K. Govind, ${ }^{3}$ and Chun-Fang $\mathbf{W u}{ }^{1}$ \\ ${ }^{1}$ Department of Biological Sciences, University of lowa, lowa City, lowa 52240, ${ }^{2}$ Department of Physiology, University of \\ Toronto, Toronto, Ontario M5S 1A8, Canada, and ${ }^{3}$ Life Sciences Division, Scarborough College, University of Toronto, \\ Scarborough, Ontario M1C 1A4, Canada
}

\begin{abstract}
Mutations of the genes rutabaga (rut) and dunce (dnc) affect the synthesis and degradation of cAMP, respectively, and disrupt learning in Drosophila. Combined ultrastructural analysis and focal electrophysiological recording in the larval neuromuscular junction revealed a loss of stability and fine tuning of synaptic structure and function in both mutants. Increased ratios of docked/undocked vesicles and poorly defined synaptic specializations characterized $d n c$ synapses. In contrast, rut boutons possessed fewer, although larger, synapses with lower proportions of docked vesicles. At reduced $\mathrm{Ca}^{2+}$ levels, decreased quantal content coupled with an increase in failure rate was seen in rut boutons and reduced pair-pulse facilitation were found in both rut and $d n c$ mutants. At physiological $\mathrm{Ca}^{2+}$ levels, strong enhancement, instead of depression, in evoked release was observed in some $d n c$ and rut boutons during 10 $\mathrm{Hz}$ tetanus. Furthermore, increased variability of synaptic trans-
\end{abstract}

mission, including fluctuation and asynchronicity of evoked release, paralleled an increase in synapse size variation in both $d n c$ and rut boutons, which might impose problems for effective signal processing in the nervous system. Pharmacological and genetic studies indicated broader ranges of physiological alteration by $d n c$ and rut mutations than either the acute effects of CAMP analogs or the available mutations that affect CAMPdependent protein kinase (PKA) activity. This is consistent with previous reports of more severe learning defects in dnc and rut mutations than these PKA mutants and allows identification of the phenotypes involving long-term developmental regulation and those conferred by PKA.

Key words: Drosophila; synaptic ultrastructure; synaptic stability; variability and plasticity; neuromuscular junction; vesicle docking; learning and memory; dnc; rut; DCO; dPKA-RI
Mutational analyses of associative learning behavior in Drosophila melanogaster have identified the genes rutabaga (rut) and dunce $(d n c)$, which encode a $\mathrm{Ca}^{2+} /$ calmodulin $\left(\mathrm{Ca}^{2+} / \mathrm{CaM}\right)$-responsive adenylyl cyclase (AC) and a cAMP-specific phosphodiesterase (PDE), respectively (Dudai et al., 1976; Tully and Quinn, 1985; Chen et al., 1986; Levin et al., 1992). These enzymes regulate the synthesis and degradation of cAMP (Byers et al., 1981; Dudai et al., 1983; Livingstone et al., 1984). Previous experiments have shown abnormal habituation of the adult giant fiber escape circuit (Engel and $\mathrm{Wu}, 1996$ ), altered nerve terminal arborization in the larval neuromuscular junction (Zhong et al., 1992), and defective $\mathrm{K}^{+}$currents in both larval muscle (Zhong and Wu, 1993) and

Received Jan. 10, 2000; revised March 15, 2000; accepted March 17, 2000.

This work was supported by grants from National Institutes of Health to C.-F.W. and Natural Sciences and Engineering Research Council of Canada to C.K.G. and H.L.A. H.L.A. is a member of the Medical Research Council of Canada Group on Nerve Cells and Synapses. We thank Drs. J. Denburg, S. Karunanithi, and Martin Wojtowicz for their comments on this manuscript. We also thank Dr. Wei-Dong Yao for sharing unpublished information and for help in preparing Table 2, Mr. Peter Taft for help with stock maintenance, Marianne Hegstrom-Wojtowicz for help in constructing Figure 3, Joanne Pearce for help with electron microscopy, and Nichole Jeffries and Josie Chandler for help in the preparation of this manuscript. We also thank Drs. Ronald Davis, John Keiger, Daniel Kalderon, and Tim Tully for providing us with alleles of $d n c$, rut, $D C O$, and $d P K A-R I$.

J.J.R. and A.U. contributed equally to this work.

Correspondence should be addressed to Dr. Chun-Fang Wu, Department of Biological Sciences, University of Iowa, Iowa City, IA, 52240. E-mail: cfwu@blue. weeg.uiowa.edu.

Dr. Renger's present address: Department of Brain and Cognitive Sciences, Massachusetts Institute of Technology, Cambridge, MA 02139.

Copyright (C) 2000 Society for Neuroscience $0270-6474 / 00 / 203980-13 \$ 15.00 / 0$ dissociated embryonic neurons (Zhao and $\mathrm{Wu}, 1997)$. The results also implicate the cAMP pathway as an important factor in controlling neuronal firing patterns (Zhao and Wu, 1997) and synaptic efficacy (Zhong and Wu, 1991).

Mutational perturbations can provide structure-function insights into synaptic transmission machinery. The Drosophila larval neuromuscular junction has been well described in terms of its development (Broadie et al., 1993; Keshishian et al., 1993, 1996), ultrastructure (Atwood et al., 1993; Jia et al., 1993), and physiology (Jan and Jan, 1976a,b; Ganetzky and Wu, 1982, 1983; Zhong and Wu, 1991; Kurdyak et al., 1994; Wang et al., 1994). To investigate the particular defects caused by modifications in the cAMP cascade, we quantified electron microscopic observations and focal loose-patch recordings from single synaptic boutons at the larval neuromuscular junction. This combined approach allowed us to delineate ultrastructural defects in vesicle localization and synapse morphology associated with specific physiological alterations in spontaneous secretion, synchronicity of transmitter release, and short-term synaptic plasticity. The results extended previous whole-cell studies performed at reduced $\mathrm{Ca}^{2+}$ levels (Zhong and $\mathrm{Wu}, 1991$ ) and revealed unexpected structural and functional defects of mutant synapses at both physiological and reduced $\mathrm{Ca}^{2+}$ levels. Our focal recording demonstrated striking variability among mutant boutons, which is masked in the ensemble output of a large number of boutons in previous whole-cell recordings. Furthermore, abnormal short-term plasticity of synaptic transmission was observed in both mutants at physiological as well as reduced physiological $\mathrm{Ca}^{2+}$ concentrations, allowing a 
more relevant correlation with behavioral abnormalities. These findings indicate an important role of the cAMP cascade in maintaining stability, in addition to regulating plasticity, of synapses. Acute pharmacological treatments using membranepermeant CAMP analogs reproduced only a limited subset of the $d n c$ and rut phenotypes. Similarly, mutations that affect the activity of cAMP-dependent protein kinase A (PKA) did not induce all the synaptic defects characteristic of $d n c$ and rut. The broader range of the synaptic phenotypes seen in the $d n c$ and rut alleles might explain the more severe deficiencies of initial memory acquisition in $d n c$ and rut compared with these PKA mutants in previous behavioral studies (Dubnau and Tully, 1998).

Preliminary accounts of some of these results have been published previously (Renger et al., 1995, 1998).

\section{MATERIALS AND METHODS}

Drosophila stocks. The Drosophila melanogaster stocks used for these experiments were $y d n c^{\mathrm{M} 11} c v v / X^{\wedge} X y f / Y, y d n c^{\mathrm{M} 14} c v / X^{\wedge} X y f / Y, y d n c^{2}$ ecf, rut ${ }^{1}, P\left[\right.$ lac $Z$ rut $\left.^{1084}\right], D C O^{\mathrm{X} 4}$, and $d P K A-R I^{715}$. The visible markers, $y, c v, v, e c, f$, and a balancer chromosome, $X^{\wedge} X y f$, are described in Lindsley and Zimm (1992). The $y d n c^{\mathrm{M} 11} c v v$ and $y d n c^{\mathrm{M} 14} c v v$ stocks were from the collection of Dr. D. Mohler (University of Iowa). The strain P[lac Z rut ${ }^{1084}$ ] (Han et al., 1996) was provided by Dr. R. Davis (Baylor College of Medicine), and $y d n c^{2}$ ec $f$ was provided by Dr. J. Keiger (University of California, Davis). The $D C O$ alleles ( $\mathrm{Li}$ et al., 1995) were provided by Dr. D. Kalderon (Columbia University), and the $d P K A-R I^{715}$ strain (Goodwin et al., 1997) was from Dr. T. Tully (Cold Spring Harbor Laboratory). Because of female sterility, the $d n c^{\mathrm{M} 11}$ and $d n c^{\mathrm{M} 14}$ lines must be maintained with balancers, and only males could be used for the present experiments. The control strain for these experiments was Canton-Special wild type. Multiple alleles of independent isolates were used to control for possible unidentified second-site effects. All stocks were raised at $\sim 22^{\circ} \mathrm{C}$ on standard medium.

Electron microscopy. Larvae were dissected in HL3 saline (Stewart et al., 1994), fixed, and embedded for electron microscopy (EM) as described previously (Atwood et al., 1993). Initially, the dissected larvae were superfused for $20 \mathrm{~min}$ with a primary fixative made of 0.1 M sodium cacodylate buffer, $1 \%$ glutaraldehyde, and $4 \%$ formaldehyde. Abdominal segment 3 was isolated with parts of segments 2 and 4 attached and placed in fresh fixative for $2 \mathrm{hr}$. After fixation, the tissue was rinsed several times in the buffer solution for $30 \mathrm{~min}$ and post-fixed in buffered $1 \%$ osmium tetroxide for $1 \mathrm{hr}$. A $15 \mathrm{~min}$ rinse in buffer preceded dehydration in a graded ethanol series $(30-100 \%)$, followed by clearing in propylene oxide and embedding in plastic (EponAraldite). The plastic was cured in a $60^{\circ} \mathrm{C}$ oven for $2-3 \mathrm{~d}$, and thin sections $(50-100 \mathrm{~nm})$ were cut with a diamond knife and placed on single slot grids coated with Formvar. The tissue was double-stained with uranyl acetate and lead citrate. Electron micrographs of the innervation with a final magnification of $24,000-26,000 \times$ were used for measurements.

Muscles 6 and 7 were examined to capitalize on the extensive data previously accumulated on these two muscle fibers (Atwood et al., 1993; Jia et al., 1993). A total of 10 larvae were used: four wild type, four $d n c^{\mathrm{M} 14}$, and two $r u t^{1}$. In each of these, the nerve terminals were sampled from several different areas of muscles 6 and 7 to obtain a representative picture. A total of $183 \mu \mathrm{m}$ of serially sectioned nerve terminal was examined, $\sim 100 \mu \mathrm{m}$ for wild type, $50 \mu \mathrm{m}$ for $d n c^{\mathrm{M} 14}$, and $33 \mu \mathrm{m}$ for $r u t^{1}$.

Areas of innervation with both axons innervating muscles 6 and 7 [terminals of axons 1 and 2: Atwood et al. (1993); Kurdyak et al. (1994)] were selected for cutting serial thin sections. Data on the number and size of synaptic contacts and dense bars were obtained for 5-10 $\mu \mathrm{m}$ lengths of nerve terminals by techniques described previously for Drosophila neuromuscular terminals (Atwood et al., 1993). As in previous studies of crustacean and Drosophila synapses, a "synapse" is defined as the electron-dense presynaptic and postsynaptic membranes with uniform separation found at numerous locations on a varicosity, whereas the dense bar [an indicator of the active zone; see Meinertzhagen et al. (1998)] is the specialized region of dense projections surrounded by clustered vesicles within a synapse (Atwood et al., 1993). The volume percentages of mitochondria, clear synaptic vesicles, and subsynaptic reticulum were estimated on every fifth micrograph in a series using a lattice test system (Weible et al., 1969). An acetate sheet marked with a $1 \mathrm{~cm}$ grid was placed over the micrograph of the terminal, and the number of points falling on each component was counted and used to estimate the volume percentages.

Focal recording. Post-feeding third instar larvae were chosen for recording. At this developmental stage, larvae are prominent in size and crawl from the food onto the sides of the culture vials. Dissections were performed in modified Schneider's Drosophila culture medium (Life Technologies, Gaithersburg, MD) or $\mathrm{Ca}^{2+}$-free HL3 saline containing (in mM): $70 \mathrm{NaCl}, 5 \mathrm{KCl}, 20 \mathrm{MgCl}_{2}, 10 \mathrm{NaCHO}_{3}, 5$ Trehalose, 115 Sucrose, and 5 HEPES, pH 7.2 (Stewart et al., 1994). Larvae were then thoroughly rinsed with HL3 saline. $\left[\mathrm{Ca}^{2+}\right]_{\mathrm{o}}$ varied for different experiments as specified in each figure. Dissected larvae were viewed with differential interference contrast optics through a $40 \times$ water immersion objective on an upright Zeiss compound microscope.

Focal recording electrodes were pulled from glass capillary tubes (75 $\mu \mathrm{l}, 1.5 \mathrm{~mm}$ outer diameter; VWR, West Chester, PA) on a pipette puller (model pp-83; Narishige, Tokyo, Japan) and then polished and bent on a microforge (model de fonbrune; Aloe Scientific, St. Louis, MO) to allow a perpendicular approach to the muscle for obtaining better seal resistance. The focal recording electrodes typically had an inner diameter of 4-8 $\mu \mathrm{m}$ and were filled with HL3 solution.

All boutons recorded were from Type I terminal branches on muscle 13, of abdominal segment 3 . Their locations on the dorsal surface of muscle 13 make it easier to obtain high-resistance seals. More lateral innervation on muscles 6,7 , and 12 makes them less favorable for focal recording, although previous whole-cell recordings showed no distinct properties of neuromuscular transmission among the Type I boutons of the four muscle fibers.

The segmental nerves were severed from the ventral ganglion and stimulated with a suction electrode (10 $\mu \mathrm{m}$ inner diameter) through the cut end. Recordings were made with a loose patch-clamp amplifier (model Double Pulse Patch Clamp 8510; Zeitz Instruments, Munich, Germany) (Dudel, 1981) and stored on VCR tapes with a Pulse Code Modulator (model Neuro-Corder DR-384; Neuro Data, New York, NY). All trials contained a calibration pulse to measure the electrode series and seal resistances. These measurements were used to correct for attenuated current amplitudes at the pipette tip (Stühmer et al., 1983). Data were rejected if the seal resistance varied more than $15 \%$ throughout the experiment.

Stimuli were applied to the segmental nerve near the entry point to the body-wall muscle fibers where the branch innervating muscles 12 and 13 begins to traverse underneath muscles 6 and 7. Furthermore, the stimuli were adjusted to two to three times threshold voltage $(2-3 \mathrm{~V}$ at $0.3 \mathrm{msec})$ to ensure effective stimulation of the bouton. Spontaneous activity was also observed during the recording period. The results were not accepted if spontaneous events were not clearly registered during the experiment. For Figure 4, the quantal content was calculated by dividing the averaged amplitude of evoked excitatory junctional currents (ejcs) by the averaged amplitude of spontaneous miniature ejcs (mejcs). In a subset of wild-type $(n=13), d n c^{\mathrm{M} 11}(n=6)$, and $r u t^{1}(n=8)$ boutons, quantal content was also determined on the basis of charge transfer (using area underneath the ejc and mejc traces). Both methods yielded similar conclusions. For Figures 6, 7C, and 8, direct electrotonic stimulation was applied to nerve terminals (Ganetzky and $\mathrm{Wu}, 1982$ ). This minimized the potential contributions to variability in synaptic release from unidentified alterations in action potential shape or conduction speed in mutant axons. Electrotonic stimuli of increased duration $(1 \mathrm{msec})$ applied near the nerve entry point were adjusted until the evoked ejc amplitude saturated. cAMP, $\mathrm{N}^{6}, \mathrm{O}^{2}$-dibutyryl-, sodium salt (db-cAMP, Sigma, St. Louis, MO) and cAMP monophosphorothioate, Rp-isomer, triethylammonium salt (Rp-cAMPS, RBI, Natick, MA) were used in pharmacological experiments. After bath application, preparations were incubated for 3-10 min before recording for db-cAMP $(500 \mu \mathrm{M})$ or for $10 \mathrm{~min}$ for Rp-cAMPS $(40-50 \mu \mathrm{M})$.

Analyses were performed with the software Axograph (versions 2.0 and 3.0; Axon Instruments, Foster City, CA) on a computer (model Power Macintosh 7100/80; Apple Computer, Cupertino, CA). Time constants of focal ejc decay were fit by using the Chebyshev algorithm (Axograph 2.0 and 3.0). Values of fits were confirmed by visual inspection of curve overlay with actual records. For presentation, some traces were digitally filtered at $1 \mathrm{kHz}$. 
Table 1. Number and size of synapses and active zone dense bars of axons 1 and 2 in serial sections of larval abdominal muscles 6 and 7

\begin{tabular}{|c|c|c|c|c|c|c|}
\hline & \multicolumn{3}{|l|}{ Axon 1} & \multicolumn{3}{|l|}{ Axon 2} \\
\hline & Control & $d n c^{M 14}$ & $r t^{1}$ & Control & $d n c^{M 14}$ & $r u t^{I}$ \\
\hline Terminal length sectioned $(\mu \mathrm{m})$ & 27.28 & 23.75 & 18.88 & 44.49 & 16.80 & 12.76 \\
\hline \multicolumn{7}{|l|}{ Synapse } \\
\hline Total number & 171 & 147 & 49 & 137 & 44 & 15 \\
\hline Number/ $\mu \mathrm{m}$ of terminal & 6.3 & 6.2 & 2.6 & 3.1 & 2.6 & 1.2 \\
\hline Mean size $\pm \mathrm{SD}\left(\mu \mathrm{m}^{2}\right)$ & $0.315 \pm 0.183$ & $0.335 \pm 0.218$ & $1.000 \pm 0.854$ & $0.295 \pm 0.187$ & $0.489 \pm 0.385$ & $0.469 \pm 0.302$ \\
\hline Range $\left(\mu \mathrm{m}^{2}\right)$ & $0.034-0.778$ & $0.062-1.210$ & $0.099-3.588$ & $0.400-0.813$ & $0.012-1.629$ & $0.124-1.310$ \\
\hline Area $/ \mu \mathrm{m}$ of terminal $\left(\mu \mathrm{m}^{2}\right)$ & 1.975 & 2.077 & 2.596 & 0.909 & 1.280 & 0.551 \\
\hline \multicolumn{7}{|l|}{ Dense bars } \\
\hline Total number & 167 & 162 & 110 & 134 & 62 & 24 \\
\hline Number/synapse & 0.976 & 1.080 & 2.240 & 0.978 & 1.409 & 1.600 \\
\hline Number/ $\mu \mathrm{m}$ of terminal & 6.1 & 6.7 & 5.8 & 3.0 & 3.7 & 1.9 \\
\hline Mean length $\pm \mathrm{SD}(\mu \mathrm{m})$ & $0.175 \pm 0.065$ & $0.187 \pm 0.064$ & $0.201 \pm 0.073$ & $0.160 \pm 0.069$ & $0.181 \pm 0.079$ & $0.153 \pm 0.049$ \\
\hline Range $(\mu \mathrm{m})$ & $0.070-0.370$ & $0.070-0.445$ & $0.070-0.390$ & $0.070-0.276$ & $0.050-0.550$ & $0.075-0.255$ \\
\hline Length/ $\mu \mathrm{m}$ of terminal $(\mu \mathrm{m})$ & 1.073 & 1.270 & 1.173 & 0.482 & 0.668 & 0.287 \\
\hline \multicolumn{7}{|l|}{$\%$ of synapses with } \\
\hline 0 dense bars & 18 & 13 & 2 & 23 & 7 & 0 \\
\hline 1 dense bars & 66 & 65 & 43 & 59 & 57 & 60 \\
\hline 2 dense bars & 14 & 20 & 19 & 14 & 25 & 27 \\
\hline 3 or more dense bars & 2 & 2 & 36 & 4 & 11 & 13 \\
\hline
\end{tabular}

\section{RESULTS}

\section{Ultrastructural features in synaptic boutons of the larval neuromuscular junction}

Previous work has shown that $d n c$ and rut mutations cause defects in gross neuronal morphology, including reduced growth cone motility in cultured neurons (Kim and Wu, 1996), abnormal arborization of axonal branches of the third instar larval neuromuscular junction (Zhong et al., 1992; Schuster et al., 1996), and altered thoracic projections of mechanosensory neurons (Corfas and Dudai, 1991) and Kenyon cell fibers of the mushroom bodies (Balling et al., 1987) of adult flies. Therefore, it is important to examine whether ultrastructural abnormalities of synapses are present in these mutants. The motor terminal boutons of muscles 6 and 7 of third instar larvae have been well characterized by serial section reconstruction (Atwood et al., 1993; Jia et al., 1993), providing a solid foundation for analysis of possible cAMP cascade-induced defects at the ultrastructural level.

We analyzed both randomly sampled and serial EM sections of individual boutons and measured morphometric parameters for terminals of axons 1 and 2 in muscles 6 and 7 [see Materials and Methods and Atwood et al. (1993)]. Axons 1 and 2 [from neurons RP3 and 6/7b, respectively (Keshishian et al., 1993)] were analyzed in parallel (Table 1). Although these axons have some morphological differences (Atwood et al., 1993; Jia et al., 1993), defects associated with $d n c$ and rut mutants were found to be common to both axons.

General features of axons 1 and 2 in all three genotypes (control, $d n c^{\mathrm{M} 14}$, and $r u t^{1}$ ) were in accordance with those described previously (Atwood et al., 1993). For axon 1 terminals, nerve terminal varicosities were usually larger, with the surrounding subsynaptic reticulum more densely packed (Fig. 1). Also, axon 1 terminals only occasionally contained dense-core vesicles, whereas they were consistently found in axon 2 terminals (up to $1 \%$ of volume). Additionally, more mitochondria were found in axon 1 terminals (7-8\% of the volume) than in axon 2 terminals (3-4\% of the volume).

One important difference in $d n c$ synaptic terminals is that poorly defined synapses, attributable to less dense staining of synaptic membranes, were found in both single and serial sections (Fig. 1). Because preparative procedures were the same for all three genotypes, there may be a difference in membrane composition of $d n c$ synapses that alters their staining properties.

\section{Altered synaptic specializations in dnc and rut boutons}

Examination of serial and randomly selected single sections showed differences in the number and size of synaptic contact areas (Figs. 1, between short arrows, $2 A, B, D$, in close-up, between bars) among the three genotypes, as summarized in Table 1 . In boutons of both the terminals of axons 1 and 2, synapse number was calculated per unit length of terminal sectioned. Similar numbers of synapses were found in control and $d n c$, but a greater than twofold reduction was observed in rut terminals. The opposite was found for size of synaptic contact: rut synapses are approximately threefold larger than those of control and $d n c$ in Axon 1 terminals ( $p<0.0001$, Student's $t$ test). An increase in synapse size was observed in axon 2 terminals in both $d n c$ and rut. When both the number and size of synapses were taken into account, a tendency for compensation was noted in the mutants.

Table 1 provides the number of active-zone dense bars (Figs. 1, 2 , long arrows) for all genotypes. In general, rut contained more dense bars per synapse, with approximately one per synapse occurring in control and $d n c$, and nearly two in rut, consistent with the larger rut synapses. Dense bars measured $\sim 0.2 \mu \mathrm{m}$ in all genotypes.

A hallmark of the mutant ultrastructural features was the variability in the size of synaptic contacts. As shown in Table 1, the range and SD for both $d n c$ and rut synapse area in axon 1 terminals were much greater than those observed in controls. The 

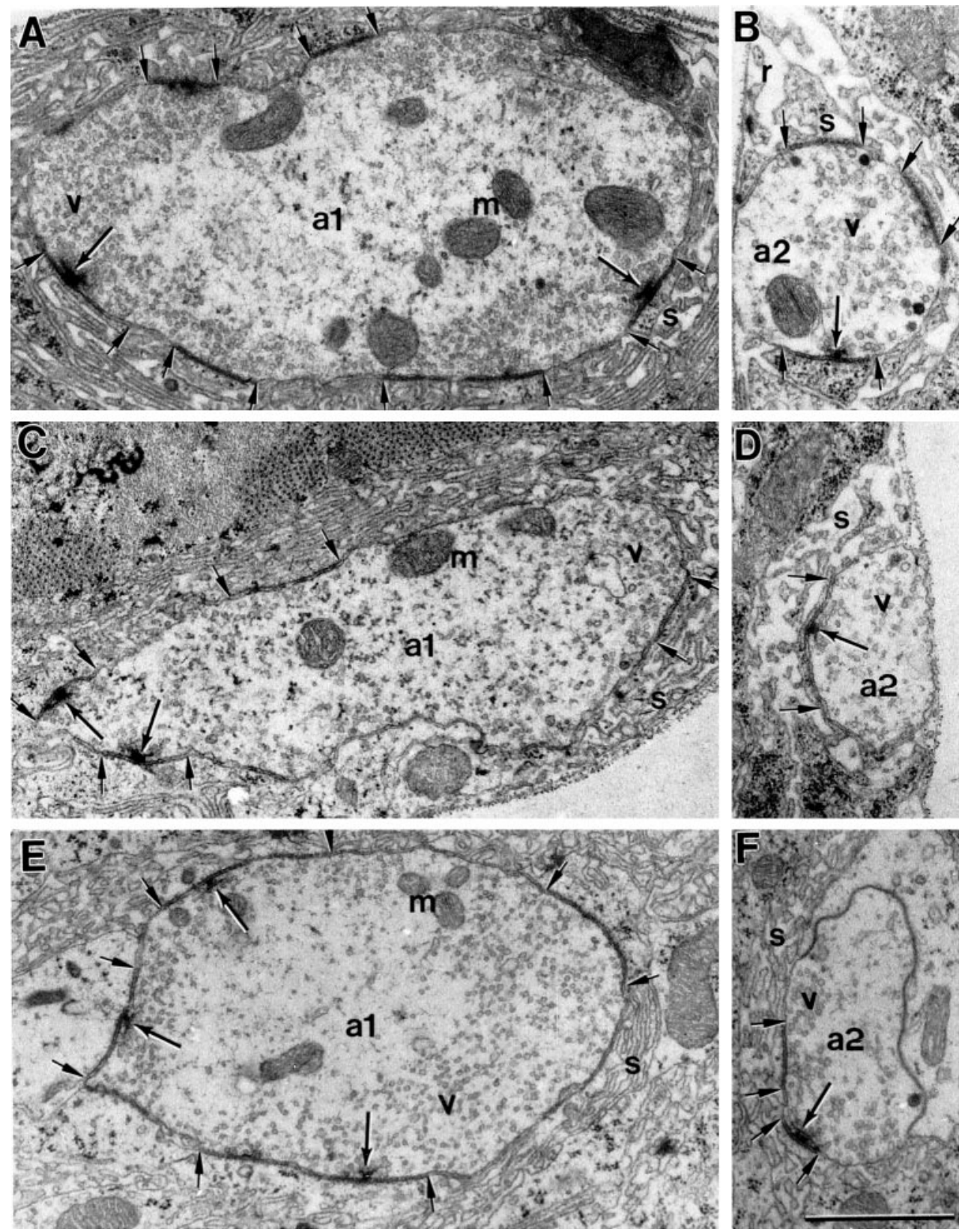

Figure 1. Ultrastructural features of motor axon terminals in larval abdominal muscle 6 of mutant and wild-type Drosophila. Terminals of axon 1 (a1) and axon 2 (a2) in wild-type $(A, B), d n c^{\mathrm{M} 14}(C, D)$, and $r^{1}{ }^{1}(E, F)$ populated with clear synaptic vesicles $(v)$ and making synaptic contact (between short arrows) with subsynaptic reticulum $(s)$. Many of the synapses show presynaptic dense bars (long arrows), the putative active zones for transmitter release. For both axons, $d n c$ synapses have less dense presynaptic and postsynaptic membranes than control, whereas rut axon 1 synapses are generally larger than controls. Scale bar, $1 \mu \mathrm{m}$. same tendency was also observed for synapse size in axon 2 terminals. In contrast, the length of dense bars remained relatively constant among genotypes. These findings demonstrate that the disruption of the cAMP cascade leads to obvious ultrastructural alterations in synaptic contacts, suggesting a previously unnoticed possibility that the dynamic balance of cAMP levels may be required for the maintenance of structural integrity and stability of synapses.

\section{Docked and undocked vesicles}

In addition to synaptic structural specializations, the localization and distribution of synaptic vesicles is also important in determining synaptic function. One particularly interesting feature is the proportions of the morphologically "docked" and "undocked" vesicles at mutant synapses. Docked vesicles were defined as those contacting the EM-dense region of the presynaptic membrane, whereas undocked reserve vesicles were defined as nearby vesicles within $500 \mathrm{~nm}$ of a synaptic contact. In serially sectioned synapses, the percentage of docked vesicles was lowest in rut and highest in $d n c$ compared with control (Table 2). A large sample of randomly selected synapses from individual EM sections was also analyzed and revealed a similar trend (Table 2). The KruskalWallis one-way ANOVA indicated significant differences in the density of vesicles in the undocked reserve pool among the genotypes also (Table 2). When the ratio of the docked to undocked vesicles was plotted for 40 synapses as another means of comparison, it revealed higher values for $d n c$ and lower ones for rut, compared with control (Fig. 3). These differences emerge clearly in the cumulative frequency distribution of the ratio of docked to undocked vesicles for these three genotypes (Fig. 3D). Altered synaptic vesicle distribution found in $d n c$ and rut terminals suggests abnormal mobilization and/or release, which could disrupt physiological aspects of synaptic transmission. 


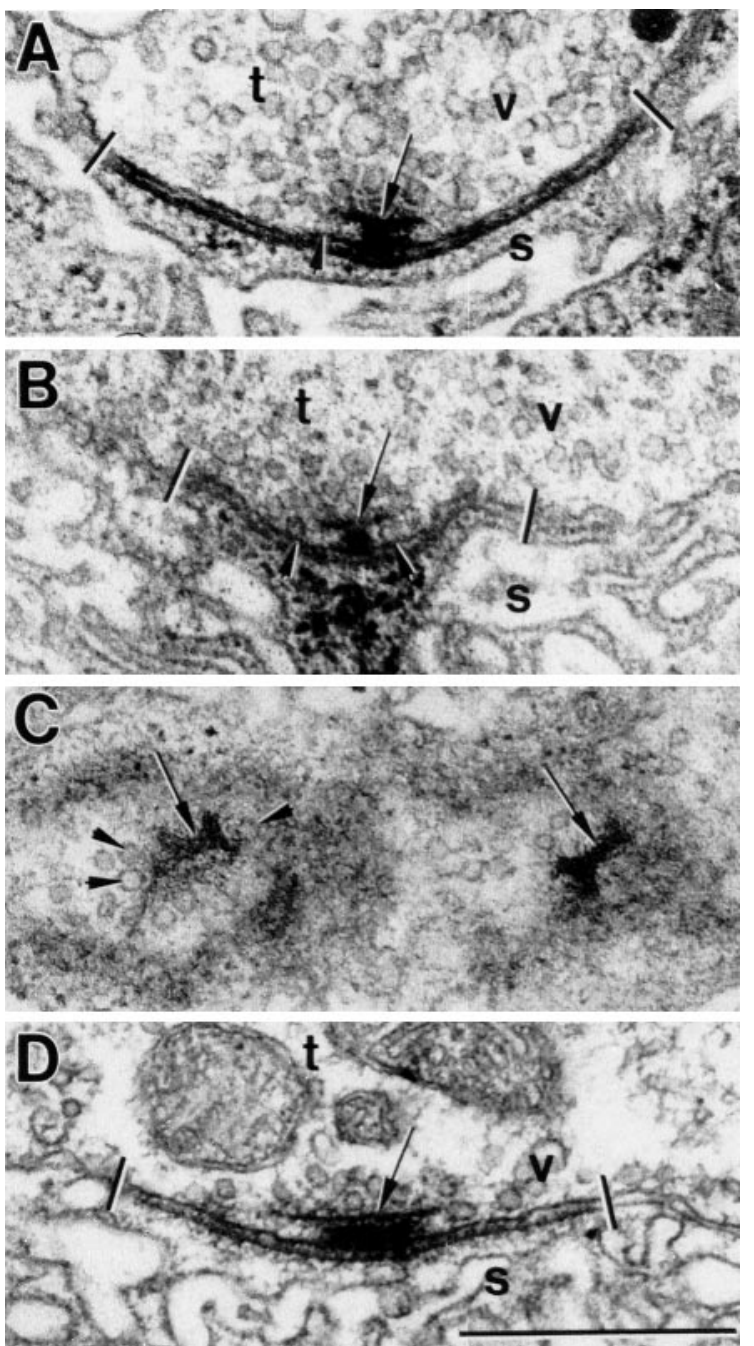

Figure 2. Presynaptic dense bars with surrounding synaptic vesicles in axon 1 nerve terminals. $A$, Cross-section through a control terminal $(t)$ populated with clear synaptic vesicles $(v)$ and showing a synaptic contact (between vertical bars) with adjacent subsynaptic reticulum $(s)$. A T-shaped dense bar (large arrow) is seen with a docked vesicle (arrowhead). $B$, Cross-section through a $d n c^{\text {M14 }}$ terminal showing a synaptic contact (between bars) that is less well defined than for controls. A presynaptic dense bar (arrow) and two docked vesicles (arrowheads) are visible. $C$, A fortuitous surface view of two presynaptic dense bars (arrows) in a $d n c$ terminal, showing the branched nature of the putative active zone with docked vesicles (arrowheads) on one arm. D, Crosssection of rut ${ }^{1}$ terminal with synaptic contact (between bars) and a presynaptic dense bar with no closely docked vesicles. Scale bar, $0.5 \mu \mathrm{m}$. Magnification 67,500×.

\section{Altered spontaneous and evoked focal synaptic currents in dnc and rut boutons}

Synaptic current amplitude

The greater variation in synaptic specializations and altered vesicle distribution described above prompted an investigation of synaptic release properties at the single-bouton level. Loose patch-clamp focal recording from individual boutons (Dudel, 1981) was performed to examine synaptic currents resulting from quantal secretion of neurotransmitter. With focal recording the local release parameters can be directly correlated with ultrastructural findings, free from confounding factors intrinsic to the whole-cell postsynaptic recordings, such as the overgrowth of axonal terminals and increases in bouton numbers seen in $d n c$
(Zhong et al., 1992; Schuster et al., 1996) and homeostatic compensation between changes in bouton numbers and synaptic release properties [see, for example fasII (Stewart et al., 1996)]. Boutons in Type I axonal branches on muscle 13 of abdominal segment 3 were recorded. These terminals are readily accessible on the dorsal surface of the muscle, favoring high-resistance seals between the muscle and electrode tip. These properties are not seen in other frequently described muscles 6, 7, and 12, although all have similar properties of whole-cell ejcs (Zhong and $\mathrm{Wu}$, 1991; Wang et al., 1994) and synaptic ultrastructure (Atwood et al., 1993).

Even at the unstimulated individual boutons, defects could be detected in the spontaneous quantal release of neurotransmitter that underlie mejcs. The spontaneous mejcs (Fig. 4A), after correction for attenuation attributable to seal resistance (see Materials and Methods), yielded no significant difference in the peak amplitude between mutant and control mejcs (Fig. 4B), but the frequency of spontaneous release for control larvae was almost twice that observed in $d n c^{\mathrm{M} 11}, d n c^{2}, r u t^{1}$, and $r u t^{1084}$ mutants. This reduction in frequency was statistically significant in all mutant genotypes at $0.5 \mathrm{~mm}\left[\mathrm{Ca}^{2+}\right]$ (Fig. $4 B$ ).

Sample traces of ejcs evoked by nerve stimulation in $0.5 \mathrm{~mm}$ $\left[\mathrm{Ca}^{2+}\right]$ are shown for the three genotypes in Figure $4 C$. The high frequency of failures and reduced release were evident for rut terminals, as shown by the quantal fluctuation in the response. Figure $4 D$ summarizes results from multiple $d n c$ and $r u t$ alleles at $0.5 \mathrm{~mm}\left[\mathrm{Ca}^{2+}\right]$ to compare their evoked release in terms of mean quantal content, or the average number of quanta released per stimulus (determined by dividing the average amplitude of evoked ejcs by the average amplitude of spontaneous mejcs; see Materials and Methods). There is a significant reduction in the mean quantal content for the rut but not $d n c$ alleles. Boutons in $d n c^{\mathrm{M} 11}$ and $r u t^{1}$ mutants were also studied at higher $\left[\mathrm{Ca}^{2+}\right](1.0$ and $1.5 \mathrm{~mm}$ ) (Fig. $4 F$ ). The peak amplitude of evoked ejcs was consistently lower in $\mathrm{rut}^{1}$ than control for all $\left[\mathrm{Ca}^{2+}\right]$ tested, whereas it was not different from control boutons at $1.0 \mathrm{~mm}$ but decreased at $1.5 \mathrm{~mm}$ in $d n c^{\mathrm{M} 11}$.

\section{Synaptic current time course}

In addition to lower frequencies of occurrence, we observed that the mutant spontaneous mejcs had apparently longer decay time course than control at different $\left[\mathrm{Ca}^{2+}\right]$ (Fig. $4 A, E$ ). As a firstorder approximation, we fit the falling phase of mejc traces with single exponential decays. It is possible that the increase in mejc decay time for the mutants is partly caused by defective reuptake mechanisms of the neurotransmitter (cf. Jan and Jan, 1976b) or altered desensitization of the postsynaptic glutamate receptors (Dudel et al., 1992; Hechmann et al., 1996) in these mutants.

The prolonged decay of spontaneous mejcs is expected to increase the decay time of evoked currents. To a first-order approximation, the time constant fit to the decay of evoked ejcs for $d n c$ and rut was indeed increased (Fig. $4 E, F$ ). Figure $4 E$ compares traces of evoked ejcs (bottom traces in each panel) with those of spontaneous mejcs (top traces) at 0.7 and $1.5 \mathrm{~mm}$ external $\left[\mathrm{Ca}^{2+}\right]$. We noticed a $\mathrm{Ca}^{2+}$-dependent increase in decay times of the evoked ejcs, which was not expected from the $\mathrm{Ca}^{2+}$ independent increase in mejc decay. Increasing $\left[\mathrm{Ca}^{2+}\right]$ from 1.0 to $1.5 \mathrm{~mm}$ led to an increase in the decay time constant in both rut and $d n c$ but not in control boutons (Fig. $4 F$ ), suggesting possible dispersion of quantal release in evoked ejcs.

Indeed, both $d n c$ and rut mutations caused substantially increased variation in the temporal control of release. Broadened or 
Table 2. Distribution of synaptic vesicles from serial or single sections of axon 1

\begin{tabular}{|c|c|c|c|c|c|c|}
\hline \multirow[b]{3}{*}{ Specimens } & \multicolumn{3}{|c|}{ Serial sections } & \multicolumn{3}{|c|}{ Selected single sections } \\
\hline & \multirow[b]{2}{*}{$\begin{array}{l}\text { Number of } \\
\text { synapses }\end{array}$} & \multicolumn{2}{|l|}{ Vesicles } & \multirow[b]{2}{*}{$\begin{array}{l}\text { Number of } \\
\text { synapses }\end{array}$} & \multicolumn{2}{|l|}{ Vesicles } \\
\hline & & $\begin{array}{l}\text { Per } \mu \mathrm{m}^{3} \text { of reserve } \\
\text { pool volume }\end{array}$ & $\begin{array}{l}\% \text { docked per } \mu \mathrm{m}^{2} \\
\text { synapse area }\end{array}$ & & $\begin{array}{l}\text { Per } \mu \mathrm{m}^{2} \text { of reserve } \\
\text { pool area }\end{array}$ & $\begin{array}{l}\% \text { docked per } \\
\text { section }\end{array}$ \\
\hline Control & 10 & $1998 \pm 43$ & $9.8 \pm 1.0$ & 40 & $155 \pm 5$ & $9.6 \pm 0.7^{*}$ \\
\hline$d n c^{M 14}$ & 10 & $3079 \pm 404^{*}$ & $13.3 \pm 0.5^{*}$ & 40 & $173 \pm 8$ & $13.3 \pm 0.6^{*}$ \\
\hline$r t^{1}$ & 10 & $1844 \pm 198$ & $7.8 \pm 1.4$ & 41 & $162 \pm 8$ & $5.8 \pm 0.5^{*}$ \\
\hline
\end{tabular}

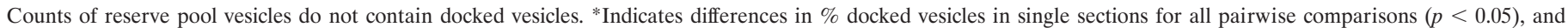

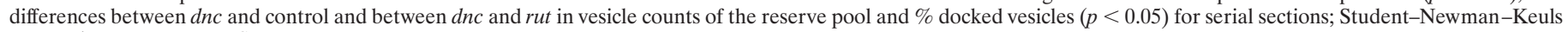
test. Values are mean \pm SD.
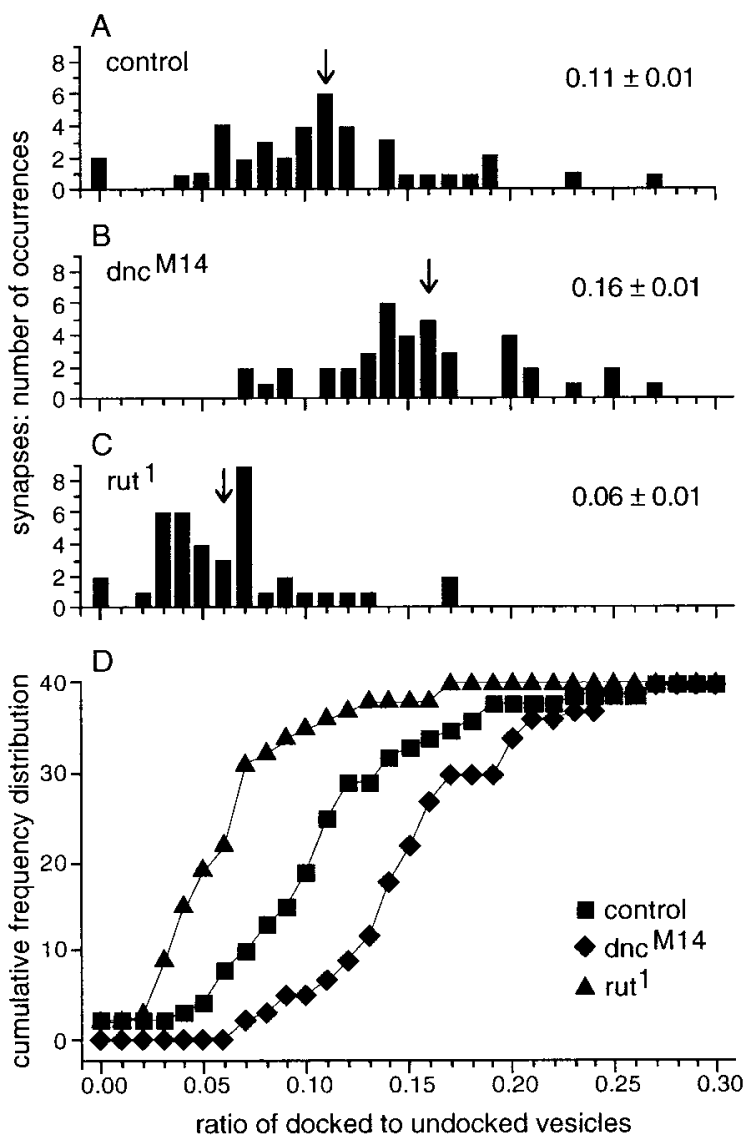

Figure 3. Comparison of ratios of docked to reserve pool synaptic vesicles in mutant and wild-type synapses. $A-C$, Histograms display 40 individual synapses in control $(A), d n c^{\mathrm{M} 14}(B)$, and $r u t^{1}(C)$, according to the frequency of occurrence of the ratio of docked to undocked vesicles. The mean ratios (denoted by arrows) and SEM are given (also see Table 2). $D$, Cumulative frequency distributions of ratios of docked to undocked vesicles in 40 synapses of the three genotypes. Differences between the distributions of $d n c$ and control, as well as rut and control, are statistically significant ( $p<0.01$, Kolmogorov-Smirnov two-sample test).

multiple peaks occurred in evoked ejcs in both $d n c$ and rut (Fig. 4, $C, 0.5 \mathrm{~mm}$, and $E, 0.7$ and $\left.1.5 \mathrm{~mm}\left[\mathrm{Ca}^{2+}\right]\right)$. When a large number of traces of evoked ejcs for individual mutant boutons are averaged, slower time to peak becomes evident (data not shown).

\section{Defective temporal regulation of evoked release in dnc and} rut boutons

In addition to the control of the amount of release, synchronicity of quantal release on nerve stimulation is important to reliable signal processing. To quantify the effect of altering the cAMP cascade on the temporal regulation of release, we examined a large number of boutons for each genotype and plotted the SD of time to peak of electrotonically evoked ejcs (Fig. 5). Direct electrotonic stimulation of the boutons was used to minimize the contribution from potential variability in action potential shape or conduction speed in mutant nerve terminals (see Materials and Methods). Greatly increased variability in peak time was observed for both dnc and rut ejcs, suggesting that either an increase or decrease in the cAMP concentration will lead to poor temporal control of release (see further pharmacological and genetic analyses below). The observed lack of synchronicity of release (Fig. 5) attributable to temporal dispersion of quanta (Fig. 4C, E) may be a contributing factor to the increase in decay time of evoked currents in the mutants, which exceeds the decay time observed for spontaneous quantal currents.

\section{Defective short-term synaptic plasticity in cAMP-altered boutons}

Interestingly, the greater asynchronicity in release (Fig. 5) within individual $d n c$ and rut boutons paralleled a greater variability in short-term plasticity. In a series of experiments, pair-pulse and tetanus stimuli were used to examine the activity-dependent modification of vesicle release at different $\mathrm{Ca}^{2+}$ concentrations.

\section{Pair-pulse facilitation and depression}

The twin-pulse stimulation protocol has been widely used to characterize presynaptic changes during short-term facilitation (Zucker, 1989; Zhong and Wu, 1991; Byrne and Kandel, 1996; Fisher et al., 1997). Sample traces for $40 \mathrm{msec}$ interpulse intervals are shown for 0.7 and $1.5 \mathrm{~mm}$ external $\left[\mathrm{Ca}^{2+}\right]$ in Figure $6 A$. In control boutons, facilitation was most prominent at low $\left[\mathrm{Ca}^{2+}\right]$, but facilitation changed to depression when $\left[\mathrm{Ca}^{2+}\right]$ was increased beyond $1.0 \mathrm{~mm}$. At $0.5 \mathrm{~mm}$, the twin-pulse protocol produced more than $100 \%$ facilitation in control boutons $\left(\left[I_{2}-I_{1}\right] / I_{1}>1.0\right)$ (Fig. 6B), but in both the $d n c$ and rut boutons the level of facilitation was severely reduced (Fig. $6 B, 0.5 \mathrm{~mm}$ ), similar to that previously reported in whole-cell results (Zhong and $\mathrm{Wu}, 1991$ ). Boutons of $d n c^{\mathrm{M} 11}$ larvae showed a strong depression at $0.7 \mathrm{mM}$, at which concentration controls showed facilitation (Fig. 6B). A shift to synaptic depression occurred in $r u t^{1}$ between 0.7 and 1.0 $\mathrm{mm}\left[\mathrm{Ca}^{2+}\right]$, similar to controls. Apparently, abnormal regulation of transmitter release in $d n c$ and rut boutons leads to a departure from the normal relationship in which physiological conditions that reduce transmitter release are associated with enhanced facilitation, and conditions that promote greater release cause synaptic depression (compare Figs. $4 F$ and $6 B$ ). 
A

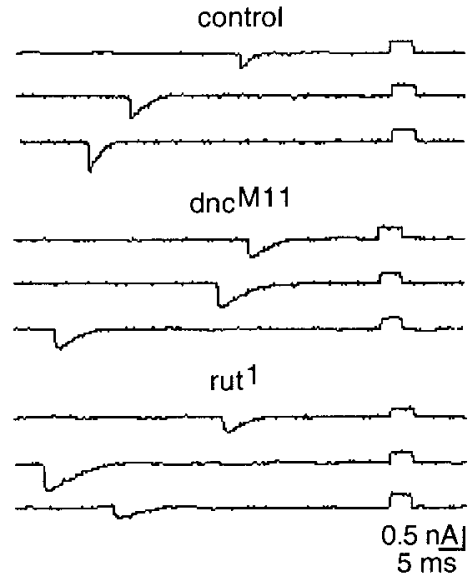

B

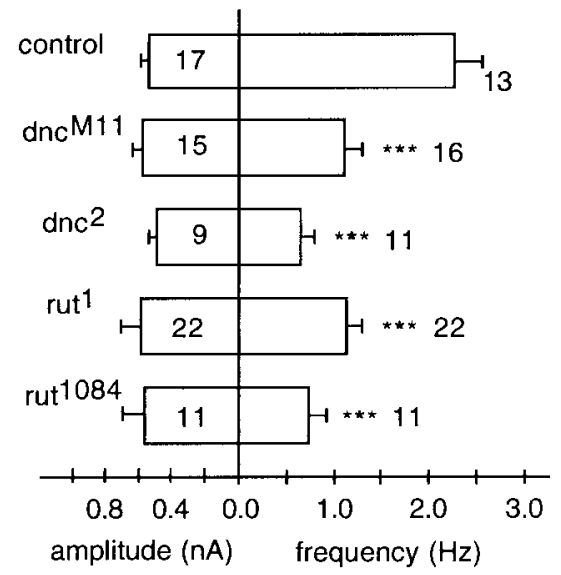

C
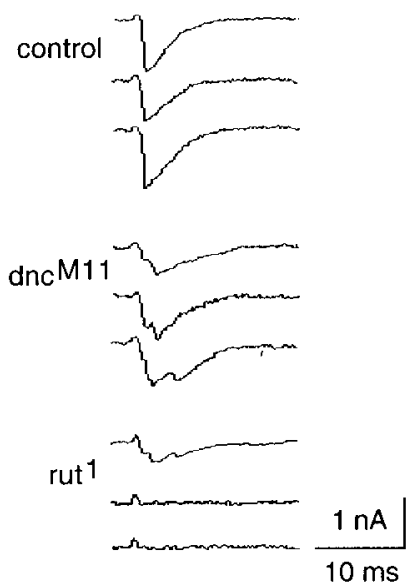

D

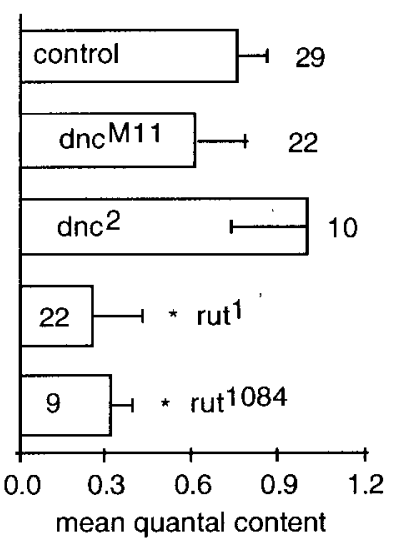

E

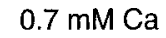

control

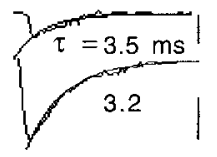

$\mathrm{dnc}^{\mathrm{M} 11}$

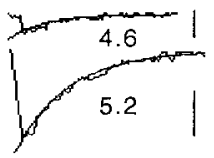

$r u t^{1}$

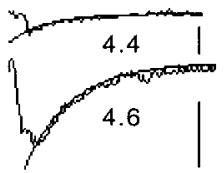

$\mathbf{F}$
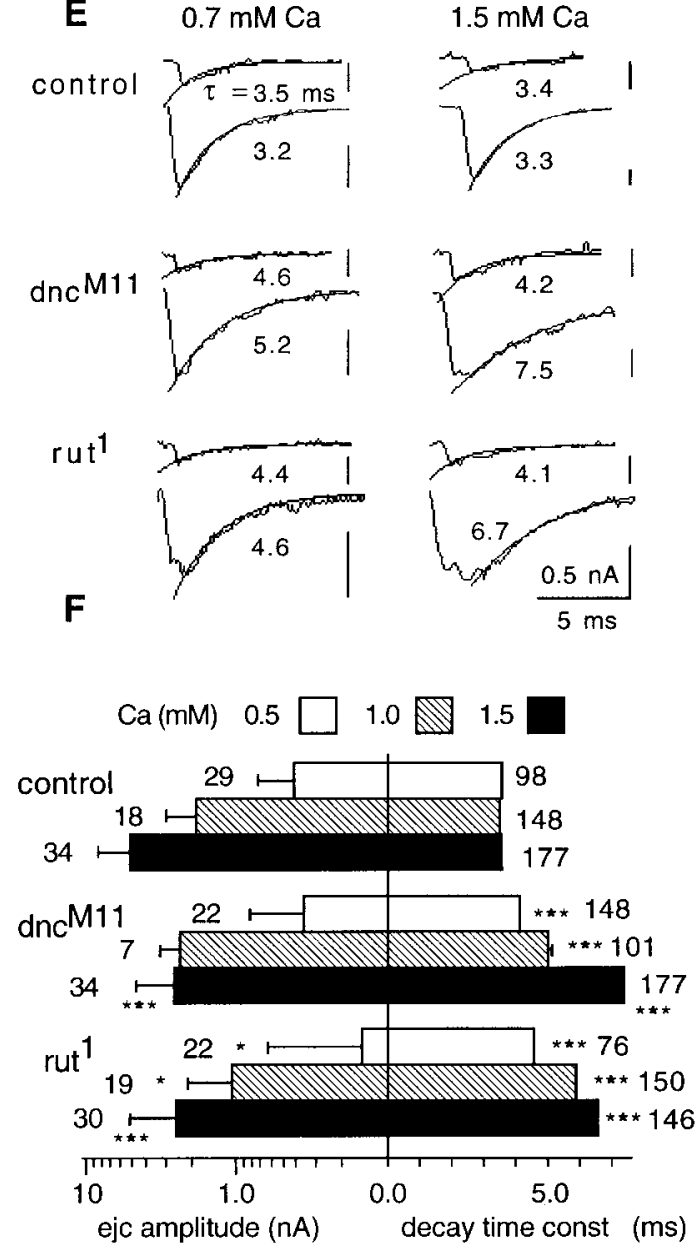

Figure 4. Focally recorded spontaneous mejcs and evoked ejcs from control, dnc, and rut boutons. A, Selected traces of focal mejcs (downward deflections) at $0.5 \mathrm{mM} \mathrm{Ca}^{2+}$ with calibration pulses (upward square pulses) that were used to correct for attenuation caused by leakage in the loose patch-clamp measurements. $B$, Amplitude and frequency histograms of spontaneous mejcs at $0.5 \mathrm{~mm} \mathrm{Ca}^{2+}$. The peak current amplitudes (left histogram) of spontaneous events were unchanged, but their frequency of occurrence (right histogram) was reduced significantly in both dnc and rut mutant boutons (mean \pm SEM from the number of boutons indicated). $C$, Selected traces of evoked ejcs at $0.5 \mathrm{mM} \mathrm{Ca}^{2+}$. Altered release properties in mutant boutons are indicated by transmission failures in rut and dispersion of release in both $d n c$ and rut. $D$, Mean quantal content of evoked ejcs at $0.5 \mathrm{~mm} C \mathrm{a}^{2+}$ for each genotype (see Results). Note reduced vesicle release from rut boutons (mean \pm SEM from the number of boutons indicated). $E$, Increase in decay time of mejcs and rise and decay time of evoked ejcs at 0.7 and $1.5 \mathrm{mM} \mathrm{Ca}^{2+}$ in $d n c$ and rut boutons. Selected individual traces of spontaneous mejcs (top traces of each genotype) and evoked ejcs (bottom traces of each genotype) are shown with an overlaid exponential decay with time constants given (see Materials and Methods). Asynchronized release in $d n c$ and rut boutons is indicated by multiple peaks in some evoked traces. $F$, Amplitude and decay time histograms of evoked ejcs at different $\mathrm{Ca}^{2+}$ concentrations. The amplitude of ejcs at $0.5,1.0$, and $1.5 \mathrm{mM} \mathrm{Ca}^{2+}$ is presented in log scale for the number of control, $d n c^{\mathrm{M} 11}$, and $r u t^{1}$ boutons indicated (left histogram). Error bars represent mean \pm SD. Note the significantly smaller mean and larger SD at different $\mathrm{Ca}^{2+}$ concentrations in rut boutons. The decay time constants were determined from the number of mejcs $\left(0.5 \mathrm{~mm} \mathrm{Ca}^{2+}\right)$ and evoked ejcs $\left(1.0\right.$ and $\left.1.5 \mathrm{mM} \mathrm{Ca}^{2+}\right)$ indicated, which was pooled from three to five boutons (right histogram). Error bars represent mean \pm SEM. Note that the decay time was longer in mutant boutons, which was further enhanced at higher $\mathrm{Ca}^{2+}$ concentrations. ${ }^{*} p<0.05$; ${ }^{* *} p<0.01 ;{ }^{* * *} p<0.001$; Student's $t$ test paired with control. For this and all of the following Figures, only Type I boutons from muscle 13 in abdominal segment 3 were used for recording at room temperature.

Previous whole-cell studies demonstrated that synaptic augmentation and post-tetanic potentiation are modified in $d n c$ and rut neuromuscular junctions differently at reduced $\mathrm{Ca}^{2+}$ levels (Zhong and Wu, 1991; Davis et al., 1996). To investigate the alteration in the ratio of docked to undocked vesicles on synaptic depression at physiological $\mathrm{Ca}^{2+}$ levels, we applied 10 $\mathrm{Hz}$ tetanus sustained for $5 \mathrm{~min}$ (Fig. 7). To minimize the potential complication of altered excitability in $d n c$ and rut neurons (Zhao and $\mathrm{Wu}, 1997)$, direct electrotonic stimulation of boutons was applied (see Materials and Methods). In control boutons, the ejcs measured in $1.5 \mathrm{~mm}\left[\mathrm{Ca}^{2+}\right]$ invariably showed depression, as indicated in the averaged response from nine boutons (Fig. 7A). Individual boutons of different output levels nevertheless displayed a similar tendency of depression as indicated by the closely grouped normalized plot (Fig. 7B). Surprisingly, the averaged responses from $d n c$ and $r u t$ boutons displayed enhanced release during the tetanic period at $1.5 \mathrm{~mm}$ $\left[\mathrm{Ca}^{2+}\right]$. The enhancement seen in the two mutants may be derived from alterations in different cellular mechanisms because contrasting phenotypes in vesicular distribution and the level of evoked release were observed in the two mutants (Figs. 3, 4; Tables 1, 2). 
A

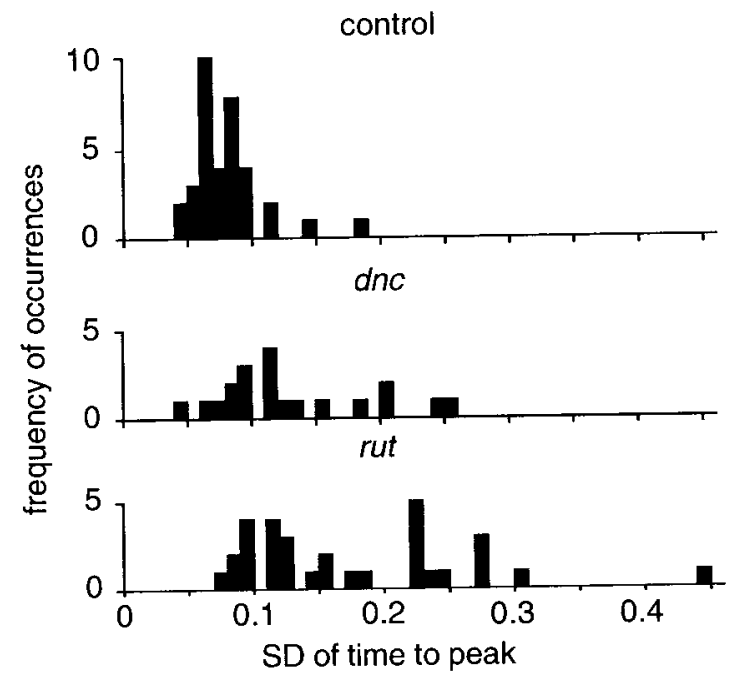

B
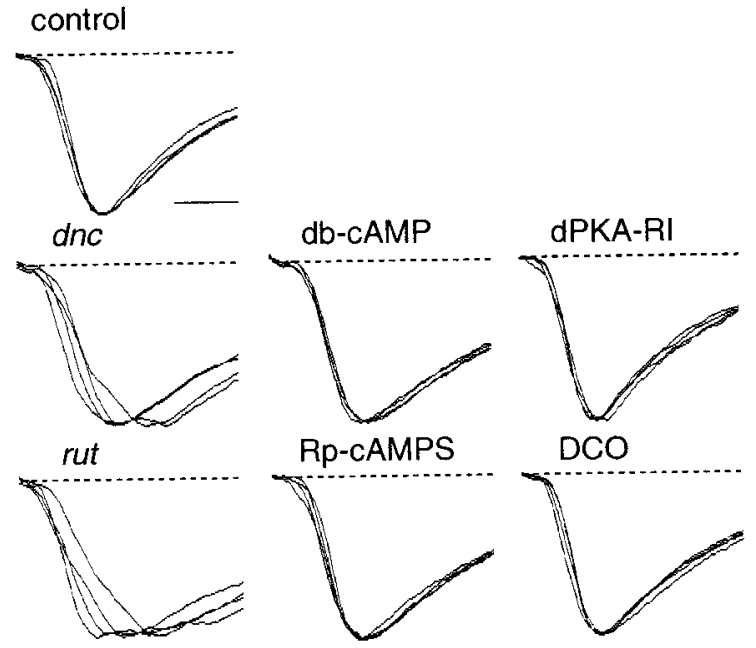

Figure 5. Increased variability in time to peak of evoked ejcs in $d n c$ and rut boutons. $A$, The distributions of SD of the peak time of evoked ejcs are shown in the histograms (number of control boutons, $d n c^{\mathrm{M} 11}$ boutons, and $r u t^{1}$ boutons is 35,20 , and 31 , respectively.). Direct electrotonic stimulation of the boutons was used to minimize the potential contribution from variation in action potential shape or conductance speed in mutant nerve terminals (see Materials and Methods). Together with the occurrence of ejcs with multiple peaks (Figs. $4 C, 5 A$ ), the spread of time-to-peak distributions for mutant boutons suggests increased temporal dispersion of quantal release. $B$, Variability in time to peak is evident in ejcs from $d n c$ and rut boutons but not from $d P K A-R I$ and $D C O$ mutant boutons or control boutons treated with db-cAMP and Rp-cAMPS. Four consecutive sample traces are normalized and superimposed for each genotype. Acute bath application of cAMP analogs, db-cAMP (500 $\mu \mathrm{M})$, or Rp-cAMPS $(40 \mu \mathrm{M})$ to the control boutons failed to mimic this phenotype of $d n c$ and rut (see Results). Little variability in time to peak was observed in the PKA mutant, $d P K A-R I$ (regulatory subunit), and $D C O$ (catalytic subunit), as in control (see Results), indicating that other downstream elements of the cAMP pathway are involved in generating the dispersal of transmitter release.

\section{Altered tetanus response and increased variability among dnc and rut boutons}

On close examination of the individual boutons, normalized responses from $d n c^{\mathrm{M} 11}$ and $r u t^{1}$ fell into two groups, one showing facilitation at $10 \mathrm{~Hz}$ and the other depression. The general tendency in rut was that boutons with initially greater output showed depression, whereas facilitation was demonstrated in those with
A
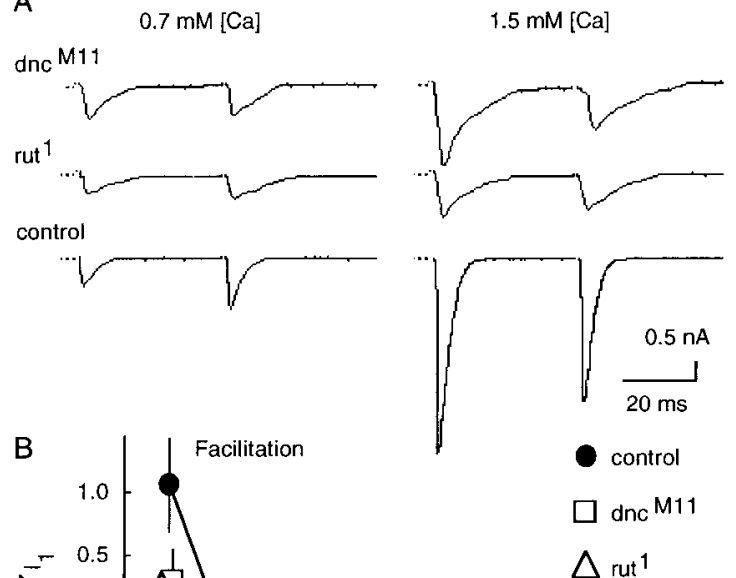

control $\square$ dnc $M 11$

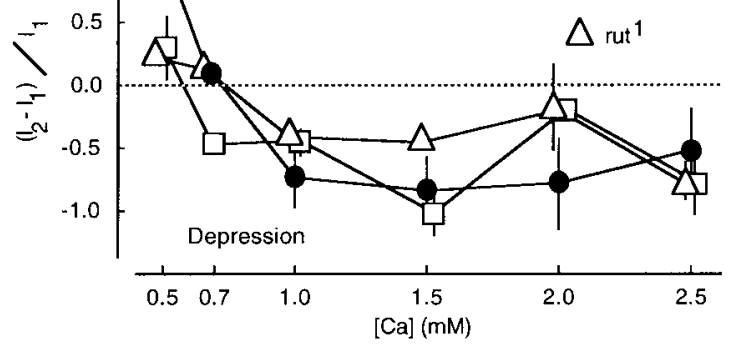

C

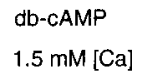

Rp-cAMPS

$1.5 \mathrm{mM}[\mathrm{Ca}]$
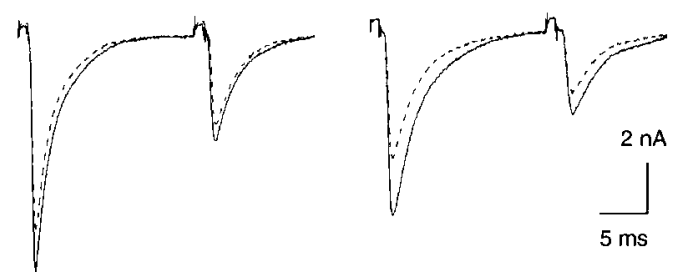

Figure 6. Altered pair-pulse facilitation in $d n c$ and rut. A, Averaged sample traces of ejc responses in pair-pulse experiments at 0.7 and $1.5 \mathrm{~mm}$ external $\left[\mathrm{Ca}^{2+}\right]$ with a $40 \mathrm{msec}$ interpulse interval. Control traces show synaptic facilitation at $0.7 \mathrm{~mm}$ and depression in $1.5 \mathrm{~mm}$. Same is true for $r u t$, but not $d n c$, traces. Traces are average of five sweeps. $B$, Average levels of synaptic facilitation and depression at different external $\left[\mathrm{Ca}^{2+}\right]$. Control boutons show greater levels of facilitation than either $d n c$ or rut boutons at $0.5 \mathrm{mM} \mathrm{Ca}^{2+}$. Rare outliers $(<5 \%)$ were excluded to show central tendencies. Error bars are SEM. The sample size for each data point ranges from 4 to 10 boutons. For each bouton, 50 trials were averaged. $C$, Bath application of cAMP analogs to control boutons reduced ejc amplitude but did not affect the pair-pulse plasticity. Averaged traces from four consecutive sweeps are shown for two pair-pulse experiments with an interpulse interval of $20 \mathrm{msec}$. The ejc responses after application of db-cAMP $(500 \mu \mathrm{M})$ and Rp-cAMPS $(40 \mu \mathrm{M})$ are shown in dotted lines. The amplitude of ejcs was reduced, but pair-pulse depression was not affected by these drugs at $1.5 \mathrm{~mm}$ external $\left[\mathrm{Ca}^{2+}\right]$ (see Results).

lower initial output (compare plots of normalized and absolute ejc amplitude in Fig. $7 B$ ). In contrast, depression or facilitation could not be predicted from the initial level of release from $d n c$ boutons (compare Fig. $7 B$ ).

The variability in response to tetanus among mutant boutons was paralleled by increased variation in output level and the temporal instability in response amplitude during repetitive stimulation. As shown in superimposed traces in Figure $7 A$, taken from a single bouton's responses before tetanus (segment $a$ ), the stability is reduced in the mutants. This is supported by the increased values of coefficient of variation (CV) of ejc amplitudes of individual boutons from each mutant observed before tetanus 

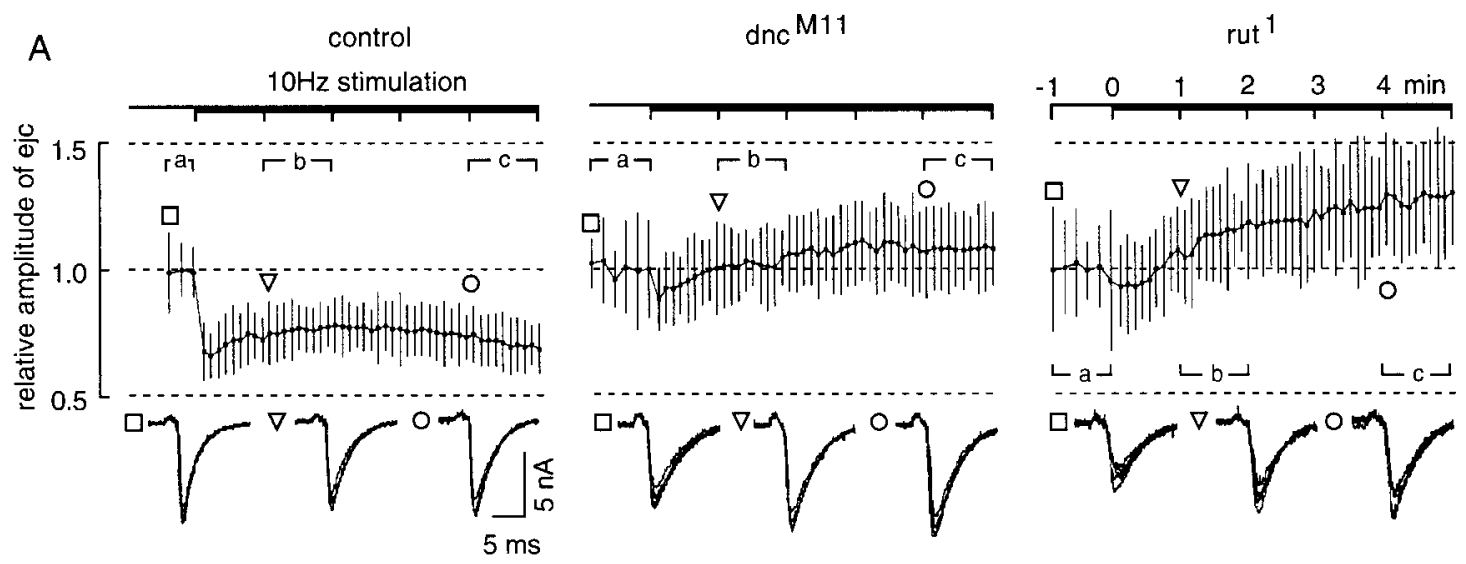

B
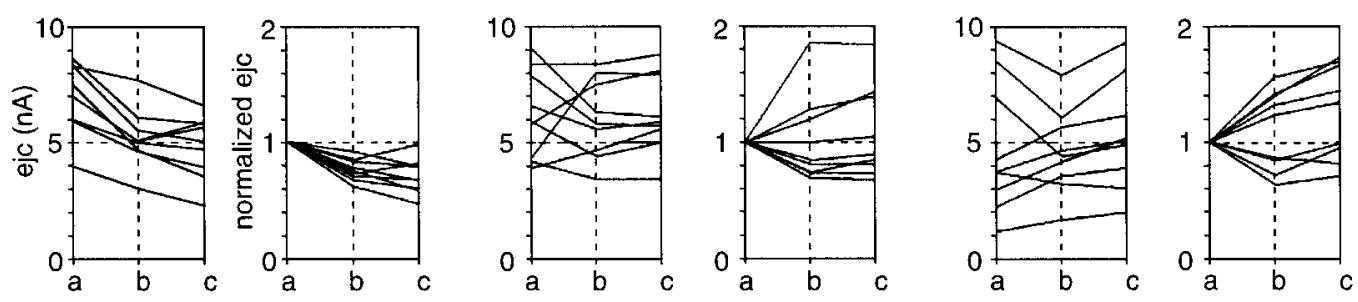

Figure 7. Altered short-term plasticity and increased variability among $d n c$ and rut boutons revealed by $10 \mathrm{~Hz}$ tetanus at a physiological $\mathrm{Ca}{ }^{2+}$ level. Test stimuli were first delivered at $0.5 \mathrm{~Hz}$ before the application of $10 \mathrm{~Hz}$ tetanus of the same stimulus strength for $5 \mathrm{~min}$ at $1.5 \mathrm{~mm} \mathrm{Ca}{ }^{2+}$. Nine individual boutons were sampled from each of the three genotypes (control, $d n c^{\mathrm{M} 11}$, and $r u t^{1}$ ) to compare their responses during tetanus. Direct electrotonic stimulation of the nerve terminal was used to minimize the potential variation in action potential shape and conduction in mutant axons (see Materials and Methods). $A$, Time course of ejc amplitude change during $10 \mathrm{~Hz}$ repetitive stimulation. The ensemble mean and SD of ejc amplitude change during tetanus are plotted for comparison among the three genotypes. The ejc amplitude for each bouton was first normalized to the average amplitude of responses to the $0.5 \mathrm{~Hz}$ test pulses. The response amplitude was sampled, and the mean and SD were determined within individual time bins during test pulses (bin size $=10 \mathrm{sec})$ and $10 \mathrm{~Hz}$ tetanus $(6 \mathrm{sec})$. Five responses were collected for each bin during test pulses $(\mathrm{sampling}$ rate $=0.5 \mathrm{~Hz})$ and 20 for each bin during tetanus $(3.3 \mathrm{~Hz})$. The mean plotted for each time bin represents the mean value of the nine individual mean ejc amplitudes, and the estimate of SD (based on the SEM of the nine boutons) is given to show the extent of variation within each time bin. Representative five consecutive ejcs are shown below the graph for each genotype at three time points, indicated by square, triangle, and circle. Note increased variation and abnormal ensemble responses during tetanus in mutant boutons. In control boutons, depression occurred during $10 \mathrm{~Hz}$ stimulation. In contrast, the ensemble responses of $d n c^{\mathrm{M} 11}$ and $r u t^{1}$ boutons showed enhancement after sustained tetanus. $B$, Variation in absolute and normalized ejc amplitudes during tetanus. The mean amplitudes of ejcs were determined for individual boutons during three selected time periods as indicated $(a$, before $10 \mathrm{~Hz}$ stimulation; $b$, between 1 to $2 \mathrm{~min}$; and $c$, between 4 and $5 \mathrm{~min}$ after the onset of tetanus). The changes in absolute ejc amplitude are shown in the left panel and are replotted by normalizing the amplitude to the pretetanus level in the right panel for each genotype. Note that control boutons consistently showed depression during tetanus, whereas enhancement and depression are both observed in mutant boutons. The greatest variation in ejc amplitude was seen in rut boutons, in which depression was correlated with those of higher ejc amplitudes and enhancement with lower amplitudes. In $d n c$ boutons no correlation of ejc amplitude and synaptic depression (or enhancement) was seen.

(average value of $\mathrm{CV}=0.10,0.13$, and 0.14 for control, $d n c^{\mathrm{M} 11}$, and $r u t^{1}$, respectively, from nine boutons each). In the case of $r u t^{1}$, an increased variability in the output level was also observed before tetanus (Fig. $7 B$, mean output level $=6.9 \pm 1.5,6.2 \pm 1.8$, and $4.8 \pm 2.7 \mathrm{nA}$, mean $\pm \mathrm{SD}, n=9$, for control, $d n c^{\mathrm{M} 11}$, and $r u t^{1}$, respectively). Similarly, an increased variability in response amplitude during tetanus was found (Fig. $7 A$, see error bars). The results demonstrate greater variability in short-term plasticity among mutant boutons and greater temporal instability within each mutant bouton as a result of altered cAMP metabolism.

\section{Limited phenocopy by acute effects of cAMP analogs and by mutations affecting PKA}

Among the $d n c$ and rut phenotypes, some could be accounted for by the immediate action of cAMP level changes, whereas others may involve long-term or developmental mechanisms. To separate acute effects from long-term regulation, we applied the membrane-permeant cAMP analogs db-cAMP $(500 \mu \mathrm{M})$, to mimic the increased basal level of cAMP in $d n c$, and Rp-cAMPS, a specific nonhydrolyzable cAMP competitor $(40-50 \mu \mathrm{M})$, to inhibit PKA activity (Nguyen and Kandel, 1996) for partial mim- icry of rut. Interestingly, only a small subset of the above phenotypes was produced by drug treatment in wild-type preparations during the course of the experiments, which sometimes lasted $>30$ min beyond drug application (see Materials and Methods). There was no change in pair-pulse facilitation at $0.5 \mathrm{mM} \mathrm{Ca}^{2+}$ [before and after db-cAMP application, pair-pulse index $($ mean $\pm \mathrm{SD})=0.47 \pm 0.83$ and $0.64 \pm 1.2, n=6$; before and after Rp-cAMPS, $0.19 \pm 0.35$ and $0.22 \pm 0.49, n=6]$. Furthermore, no changes in depression were observed at $1.5 \mathrm{mM} \mathrm{Ca}^{2+}$ (Figs. 6C, 8) (before and after db-cAMP, pair-pulse index = $-0.54 \pm 0.050$ and $-0.54 \pm 0.031, n=5$; before and after Rp-cAMPS, $-0.52 \pm 0.043$ and $-0.50 \pm 0.054, n=6)$, despite a reduction of ejc amplitude induced by these analogs (Figs. $6 C, 8$ ) [before and after db-cAMP application, amplitude (mean \pm $\mathrm{SD})=8.2 \pm 1.1$ and $7.7 \pm 1.0 \mathrm{nA}, n=5, p<0.05$; before and after Rp-cAMPS, $7.8 \pm 2.0$ and $6.5 \pm 2.2 \mathrm{nA}, n=4, p<0.05]$. Furthermore, release synchronicity remained intact (Fig. 5B) [before and after db-cAMP, SD of time to peak (mean \pm SD) $=$ $0.072 \pm 0.0053$ and $0.067 \pm 0.0057 \mathrm{msec}, n=5$; before and after Rp-cAMPS, $0.081 \pm 0.016$ and $0.079 \pm 0.014 \mathrm{msec}, n=6]$, and 
A

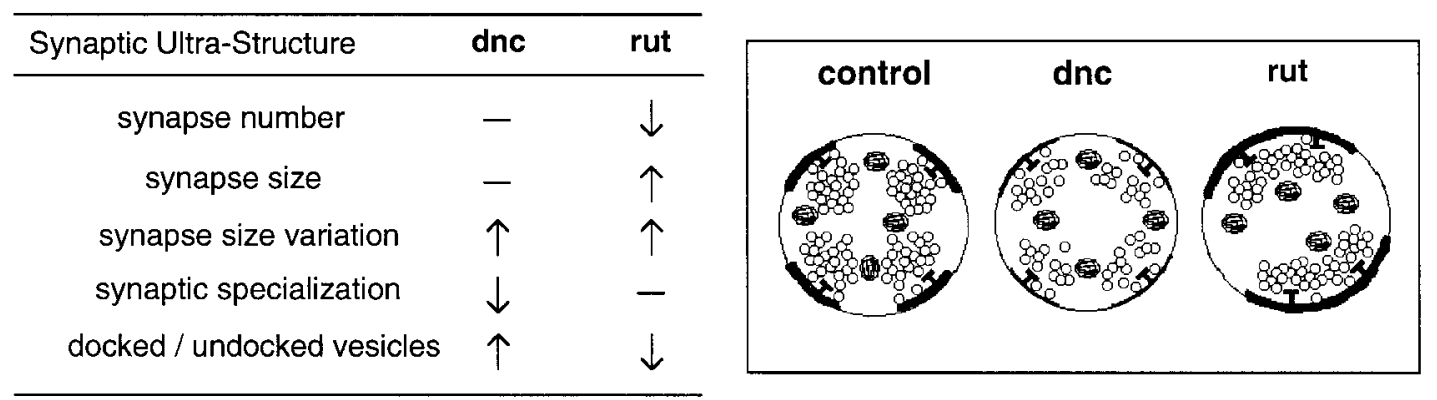

B

\begin{tabular}{ccccccc}
\hline Synaptic Physiology & dnc & db-cAMP & dPKA-RI & rut & Rp-cAMPS & DCO \\
\hline ejc amplitude & $\downarrow$ & $\downarrow$ & $\downarrow$ & $\downarrow$ & $\downarrow$ & $\downarrow$ \\
ejc amplitude variation & $\uparrow \mathrm{a}$ & - & $\uparrow w$ & $\uparrow \mathrm{aw}$ & - & $\uparrow \mathrm{aw}$ \\
release synchronicity & $\downarrow$ & - & - & $\downarrow$ & - & - \\
short-term plasticity & $\downarrow$ & - & ND & $\downarrow$ & - & ND \\
\hline
\end{tabular}

Figure 8. Summary of defects in synaptic ultrastructures and physiological properties in single boutons of Drosophila memory mutants. A, Characteristic changes in synaptic ultrastructures for $d n c$ and rut boutons. The schematic diagrams in the inset depict the alterations in synapse number, synapse size, electron-dense synaptic specializations, and ratios of docked/undocked vesicles in $d n c$ and rut boutons. As shown in the summary table, boutons in rut terminals possess fewer, albeit larger synapses with lower proportions of docked vesicles. In contrast, dnc boutons display increased proportions of docked vesicles and poorly defined synaptic specializations. In both $d n c$ and rut boutons, variation in the size of synaptic contact is greater than control. The samples were dissected in HL3 saline at a physiological $\mathrm{Ca}^{2+}$ concentration (1.5 mM) for EM sections. $B$, Synaptic physiology of mutant boutons in $d n c$, $d P K A-R I, r u t$, and $D C O$, and control boutons treated with db-cAMP and Rp-cAMPS at a physiological Ca ${ }^{2+}$ concentration (1.5 mM). Note that only the reduction of ejc amplitude in $d n c$ and $r u t$ was mimicked by PKA mutations and treatment with cAMP analogs. Asynchronicity of release in $d n c$ and rut was not reproduced by effects of either PKA mutations or cAMP analog treatments. Variation in ejc amplitude was seen in boutons of $d n c$, rut, and the PKA mutants, but not in control boutons treated with the cAMP analogs. Reduced short-term plasticity in $d n c$ and rut was based on altered pair-pulse indices, which remained intact in control boutons after treatment by cAMP analogs. These observations indicate that developmental regulation as well as additional downstream targets of cAMP are involved in some of the $d n c$ and rut phenotypes (see Discussion). $a$, Variation among boutons; $w$, variation within boutons; $N D$, not determined (because of enhanced muscle contraction after repeated stimulation in some PKA mutants).

there was no significant increase in ejc amplitude variation after treatments with both drugs (Fig. 8) [before and after db-cAMP, $\mathrm{CV}($ mean $\pm \mathrm{SD})=0.087 \pm 0.018$ and $0.076 \pm 0.014, n=5$ before and after Rp-cAMPS, $0.072 \pm 0.015$ and $0.082 \pm 0.025$, $n=4]$. In conclusion, the acute effects of both db-cAMP and Rp-cAMPS mimicked only the reduction of ejc amplitude at a physiological $\mathrm{Ca}^{2+}$ level $(1.5 \mathrm{~mm})$ observed in $d n c$ and rut. (Figs. 4, 8).

A major downstream mediator of $d n c$ and rut mutational effects is PKA, which is known to modulate a wide spectrum of protein targets. We attempted to identify genetically the $d n c$ and rut phenotypes that are mediated by PKA. The availability of mutations that alter the two subunits of PKA provided a handle to manipulate PKA activity levels (Dubnau and Tully, 1998). In particular, viable alleles could be subjected to behavioral as well as physiological characterizations to correlate their functional consequences at two different levels. Mutations of the $D C O$ gene affect the catalytic subunit of PKA, and the viable $D C O^{\mathrm{X} 4}$ allele is thus expected to mimic some of the rut phenotypes ( $\mathrm{Li}$ et al., 1995). The viable mutation, $d P K A-R I^{7 \mathrm{II}}$, modifies the regulatory subunit of PKA to render the catalytic subunit constitutively active (Goodwin et al., 1997) and therefore may mimic some of the $d n c$ phenotypes. Indeed, both $D C O^{\mathrm{X} 4}$ and $d P K A-R I^{7 \mathrm{I} 5} \mathrm{mu}-$ tations affect olfactory memory as $d n c$ and rut mutations do, although to a lesser extent (Goodwin et al., 1997; Dubnau and Tully, 1998). We found that several of the $d n c$ and rut phenotypes were not common to either $d P K A-R I$ or $D C O$ mutants (the allele identities, 7I5 and X4, are omitted hereafter for the sake of clarity). A lack of release synchronicity was not observed in either mutant, raising the possibility that this phenotype may not be mediated solely by PKA action (Figs. 5, 8) [SD of time to peak $($ mean $\pm \mathrm{SD})=0.077 \pm 0.013, n=11,0.094 \pm 0.024, n=7$, and $0.070 \pm 0.0046 \mathrm{msec}, n=5$, in control, $d P K A-R I$, and DCO, respectively). Nevertheless, the reduction in ejc amplitude at a physiological $\mathrm{Ca}^{2+}$ level $(1.5 \mathrm{~mm})$ was observed in both mutants, consistent with the decrease in $d n c$ and rut (Figs. 4, 8 [amplitude $($ mean $\pm \mathrm{SD})=5.2 \pm 3.2, n=34,2.5 \pm 1.1, n=10, p<0.05$, and $2.3 \pm 2.2 \mathrm{nA}, n=14, p<0.01$ in control, $d P K A-R I$, and $D C O$, respectively]. Furthermore, ejc amplitude variation within or among boutons seen in rut was reproduced in DCO at $1.5 \mathrm{~mm}$ $\mathrm{Ca}^{2+}$ (Fig. 8) [CV of ejc amplitudes within boutons (mean \pm $\mathrm{SD})=0.16 \pm 0.085, n=34$, and $0.34 \pm 0.25, n=13, p<0.01$; $\mathrm{CV}$ among boutons $=0.61, n=34$, and $0.96, n=14$, in control and $D C O$, respectively]. In contrast, an increase in ejc variation within boutons was observed in $d P K A-R I$ as compared with an increase among boutons found in $d n c$ (Fig. 8) [CV of ejc amplitude within $d P K A-R I$ boutons (mean $\pm \mathrm{SD})=0.25 \pm 0.066, n=$ $9, p<0.05 ; \mathrm{CV}$ among $d n c$ boutons $=0.80, n=33$ ]. These observations indicate that asynchronous release, a striking phenotype of $d n c$ and rut, may not be exclusively mediated by PKA activity. Such lack of release synchronicity may contribute to the more severe learning defects of $d n c$ and rut compared with the 
two viable PKA mutants (Li et al., 1995; Goodwin et al., 1997; Dubnau and Tully, 1998), revealing the important role of temporal control for vesicle release in effective signal processing in the nervous system.

\section{DISCUSSION}

The role of the cAMP cascade in modification of neural connectivity and synaptic strength has been demonstrated by pharmacological means in invertebrates ( $\mathrm{Hu}$ et al., 1993; Byrne and Kandel, 1996) and mammals (Nguyen and Kandel, 1996). Remarkably, an independent genetic approach has also implicated genes encoding components of the cAMP cascade in learning in Drosophila (Davis, 1996). Multiple alleles of $d n c$ and rut, which have provided valuable materials for connecting phenotypes analyzed at molecular, cellular, and behavioral levels, were isolated on the basis of poor learning performance. Furthermore, genetic perturbations of downstream elements in the cAMP pathway, such as the regulatory and catalytic subunit of PKA (dPKA-RI and $D C O$ ) and the cAMP responsive element-binding protein (dCREB2), have been subsequently shown to affect memory consolidation (Dubnau and Tully, 1998)

In this study, ultrastructural analysis combined with focal recording from individual boutons revealed novel phenotypes in synapses of Drosophila memory mutants $d n c$ and rut, including greatly increased morphological and physiological variability among synaptic boutons as well as instability of release properties within single boutons (summarized in Fig. 8). Ultrastructural observations indicate changes in synaptic specializations, and vesicle mobilization and docking, which can be evaluated in the context of physiological abnormalities. Altered short-term activitydependent plasticity, as well as reduced ejc amplitudes at physiological $\mathrm{Ca}^{2+}$ levels, delineated behaviorally relevant phenotypes in those two memory mutants.

Acute application of membrane-permeant cAMP analogs allowed an initial separation of short-term effects caused by changing cAMP levels from long-term or developmental alterations attributable to $d n c$ and rut mutations. Only some of the release properties in $d n c$ and rut can be mimicked by such pharmacological treatment in control larvae (Fig. 8), demonstrating distinct long-term influences of $\mathrm{AC}$ and PDE regulation on synaptic physiology and plasticity.

We used the mutations $d P K A-R I^{715}$ and $D C O^{\mathrm{X} 4}$ to delineate those phenotypes of $d n c$ and rut that are conferred by the PKA pathway. Again, the physiological phenotypes of $d n c$ and rut were only partially reproduced by $d P K A-R I$ and $D C O$ (Fig. 8). Notably, these PKA mutations increased ejc amplitude variation, either among or within individual boutons, mimicking $d n c$ and rut defects. Nevertheless, asynchronicity in release was not observed in these PKA mutants. This raises the possibility that some $d n c$ and rut phenotypes may involve targets besides PKA. Interestingly, both $d n c$ and rut mutants are severely deficient in both initial memory acquisition and subsequent consolidation in contrast to the relatively intact learning scores immediately after training observed in the $D C O$ and $d P K A-R I$ mutants and $C R E B$ transformants (Tully and Quinn, 1985; Yin et al., 1994; Li et al., 1995; Goodwin et al., 1997). Thus, the phenotypes that distinguish $d n c$ and rut from these PKA pathway mutants might be related to the cellular mechanisms subserving initial memory acquisition.

\section{Contributions of long- and short-term effects to synaptic function and plasticity}

Neuronal modifications underlying behavioral plasticity involve cellular mechanisms common to those of developmental plasticity (Cline, 1991; Katz and Shatz, 1996). It is important to determine the contributions of chronic or developmental effects of $d n c$ and rut to abnormal synaptic physiology and plasticity (Zhong and Wu, 1991; Zhong et al., 1992). Altered developmental regulation in both $d n c$ and rut is suggested by increased variability of synaptic contact area and by the larger size of rut synapses, possibly a homeostatic compensation for deficiencies in vesicle release. As demonstrated previously, activation of d-CREB by cAMP regulates synapse formation at the larval neuromuscular junction (Davis et al., 1996; Schuster et al., 1996). This cAMPdependent mechanism involves gene expression and is important in developmental regulation of synaptic function and growth. Some of the physiological alterations in mutant boutons may also reflect the chronic effects of disrupting cAMP metabolism (cf. Zhong and $\mathrm{Wu}, 1993$, for muscle $\mathrm{K}^{+}$currents). Acute applications of db-cAMP and Rp-cAMPS reduced ejc amplitudes but did not reproduce the altered facilitation and depression indices, increased release asynchronicity, or enhanced amplitude variation observed in $d n c$ and rut (Fig. 8).

Some physiological defects were found in both $d n c$ and rut mutants (Fig. 8) despite the opposite effects on cAMP metabolism, consistent with previous findings that both mutants show similar deficiencies in memory decay (Tully and Quinn, 1985), neuronal firing patterns (Zhao and $\mathrm{Wu}, 1997)$, and growth cone motility (Kim and $\mathrm{Wu}, 1996)$. Aside from the contrasting differences in response to pair-pulse and tetanic stimulation seen in $d n c$ and rut boutons (Figs. 6, 7), reduced frequency of spontaneous secretion (Fig. 4B), dispersal of evoked release (Fig. 4C, E), and variability in ejc amplitude (Fig. 7) were seen in both mutants. These results suggest an optimal level for cAMP or the dynamic involvement of multiple, interactive downstream targets in the regulation of synaptic function.

\section{Regulation of vesicle mobilization, transmitter release, and activity-dependent plasticity by cAMP cascade}

Previous studies have shown significant overgrowth of axonal arbors and increased bouton numbers in $d n c$ larval neuromuscular junctions (Zhong et al., 1992; Schuster et al., 1996). In the present study, the EM results revealed abnormal vesicle localization and synaptic ultrastructure. The definition of presynaptic membrane specializations was poor in $d n c$, and both area and number of synapses were altered in rut (Fig. 8, Table 1). The altered ratio of docked to reserve vesicles (Fig. 3) implies differences in rates of vesicle mobilization and release at the synapses. Previous physiological studies (Zucker, 1989; Stevens and Tsujimoto, 1995; Kuromi and Kidokoro, 1998) imply two pools of quanta, a reserve depot and a releasable pool that corresponds to the vesicles clustered at the synaptic active zone in EM studies (Pieribone et al., 1995). The reduced number of evoked and spontaneous quanta and the decreased pair-pulse plasticity in rut synapses are consistent with the decreased ratio of docked to undocked vesicles and parallel the previous whole-cell results (Zhong and Wu, 1991; Cheung et al., 1999). It should be noted that vesicular docking is only a prerequisite for release and additional steps are involved in vesicle release (Südhof, 1995). In the case of $d n c$ synapses, some of these steps might be affected, as indicated by the poor definition of synaptic structure. Despite an increased ratio of docked vesicles, $d n c$ boutons displayed a re- 
duced frequency of spontaneous release and decreased pair-pulse plasticity (Figs. 4, 6). Notably, quantal release per bouton in $d n c$ boutons is not significantly higher than normal (Fig. 4). Thus, the higher than normal whole-cell ejcs previously reported by Z hong and $\mathrm{Wu}$ (1991) in low $\left[\mathrm{Ca}^{2+}\right.$ ] could be caused either by the increased number of boutons (Zhong et al., 1992; Schuster et al., 1996) or by the use of different salines (Jan and Jan, 1976a; Stewart et al., 1994).

\section{Increased synaptic variability in dnc and rut boutons}

Our study revealed increased ultrastructural and physiological variability, including variable synaptic contact areas (Table 1) and ejc amplitudes (Fig. 7), dispersion of evoked release (Figs. 4E, 5), and inhomogeneity in short-term plasticity (Fig. 7B). The increased variability may involve chronic regulatory mechanisms because it could not be reproduced by pharmacological treatments (Fig. 8). Furthermore, the long-term effects of $D C O^{\mathrm{X} 4}$ and $d P K A-R I^{715}$ did not mirror all the characteristic phenotypes of $d n c$ and rut (Fig. 8). This indicates that some of the chronic effects of $d n c$ and rut may not be exclusively conferred by PKA activities. It is known that cAMP can directly activate $\mathrm{K}^{+}$channels at lower concentrations (Delgado et al., 1991) and cGMP-dependent protein kinase at high concentrations (Torphy, 1994; Kurjak et al., 1999). The PKG pathway also exerts a wide range of neurophysiological effects in Drosophila (Renger et al., 1999).

This initial exploration of cAMP cascade mutants at the level of single boutons has defined new phenotypes with important functional implications. Lack of stability in synaptic output and imprecise timing in responses could decrease the signal-to-noise ratio during network signal processing. Interestingly, greater variation in amplitude and duration of transmitter release from the growing terminals of developing neurons were detected in $d n c$ and rut dissociated cell cultures by using vertebrate myocytes as a probe (Yao et al., 2000). Furthermore, cultured neurons from $d n c$ and rut mutants display considerable instability in their firing patterns (Zhao and Wu, 1997). Such variable spike frequency coding could further reduce efficiency in information processing. Increased ultrastructural and functional variability among synapses may impose additional constraints on the network for reliable signal processing. Alterations in activity-dependent conditioning of synaptic efficacy at physiological $\mathrm{Ca}^{2+}$ levels (Fig. 8) could contribute to altered circuit physiology underlying abnormal behavioral plasticity in these mutants (Engel and Wu, 1996).

\section{REFERENCES}

Atwood HL, Govind CK, Wu C-F (1993) Neuromuscular junction ultrastructure of ventral abdominal muscles in Drosophila larvae. J Neurobiol 24:1008-1024.

Balling A, Technau GM, Heisenberg M (1987) Are the structural changes in the adult Drosophila mushroom bodies memory traces? Studies on biochemical learning mutants. J Neurogenet 4:65-73.

Broadie K, Sink H, Van Vactor D, Fambrough D, Whitington PM, Bate M, Goodman CS (1993) From growth cone to synapse: the life history of the RP3 motor neuron. Development [Suppl] 227-238.

Byers D, Davis RL, Kiger Jr JA (1981) Defect in cyclic AMP phosphodiesterase due to the dunce mutation of learning in Drosophila melanogaster. Nature 289:79-81.

Byrne JH, Kandel ER (1996) Presynaptic facilitation revisited: state and time dependence. J Neurosci 16:425-435.

Chen CN, Denome S, Davis RL (1986) Molecular analysis of cDNA clones and the corresponding genomic coding sequences of the Drosophila dunce ${ }^{+}$gene, the structural gene for cAMP phosphodiesterase. Proc Natl Acad Sci USA 83:9313-9317.

Cheung US, Shayan AJ, Boulianne GL, Atwood HL (1999) Drosophila larval neuromuscular junction's responses to reduction of cAMP in the nervous system. J Neurobiol 40:1-13.
Cline HT (1991) Activity-dependent plasticity in the visual systems of frog and fish. Trends Neurosci 14:104-111.

Corfas G, Dudai Y (1991) Morphology of a sensory neuron in Drosophila is abnormal in memory mutants and changes during aging. Proc Natl Acad Sci USA 88:7252-7256.

Davis GW, Schuster CM, Goodman CS (1996) Genetic dissection of structural and functional components of synaptic plasticity. III. CREB is necessary for presynaptic functional plasticity. Neuron 17:669-679.

Davis RL (1996) Physiology and biochemistry of Drosophila learning mutants. Physiol Rev 76:299-317.

Delgado R, Hidalgo P, Diaz F, Latorre R, Labarca P (1991) A cyclic AMP-activated $\mathrm{K}+$ channel in Drosophila larval muscle is persistently activated in dunce. Proc Natl Acad Sci USA 88:557-560.

Dubnau J, Tully T (1998) Gene discovery in Drosophila: new insights for learning and memory. Annu Rev Neurosci 21:407-444.

Dudai Y, Jan YN, Byers D, Quinn WG, Benzer S (1976) dunce, a mutant of Drosophila deficient in learning. Proc Natl Acad Sci USA 73:1684-1688.

Dudai Y, Uzzan A, Zvi S (1983) Abnormal activity of adenylate cyclase in the Drosophila memory mutant rutabaga. Neurosci Lett 42:207-212.

Dudel J (1981) The effect of reduced calcium on the quantal unit current and release at the crayfish neuromuscular junction. Pflügers Arch 391:35-40.

Dudel J, Franke C, Hatt H (1992) Rapid activation and desensitization of transmitter-liganded receptor channels by pulses of agonists. In: Ion channels (Narahashi T, ed), pp 207-260. New York: Plenum.

Engel JE, Wu C-F (1996) Altered habituation of an identified escape circuit in Drosophila memory mutants. J Neurosci 16:3486-3499.

Fisher SA, Fischer TM, Carew TJ (1997) Multiple overlapping processes underlying short-term synaptic enhancement. Trends Neurosci 20:170-176.

Ganetzky B, Wu C-F (1982) Drosophila mutants with opposing effects on nerve excitability: genetic and spatial interactions in repetitive firing. J Neurophysiol 47:501-514.

Ganetzky B, Wu C-F (1983) Neurogenetic analysis of potassium currents in Drosophila: synergistic effects on neuromuscular transmission in double mutants. J Neurogenet 1:17-28.

Goodwin FS, Vecchio MD, Velinzon K, Hogel C, Russell SRH, Tully T, Kaiser K (1997) Defective learning in mutants of the Drosophila gene for a regulatory subunit of cAMP-dependent protein kinase. J Neurosci 17:8817-8827.

Han PL, Meller V, Davis RL (1996) The Drosophila brain revisited by enhancer detection. J Neurobiol 31:88-102.

Hechmann M, Parzefall F, Dudel J (1996) Activation kinetics of glutamate receptor channels from wild-type Drosophila muscle. Pflügers Arch 432:1023-1029.

Hu Y, Barzilai A, Chen M, Bailey CH, Kandel ER (1993) 5-HT and cAMP induce the formation of coated pits and vesicles and increase the expression of clathrin light chain in sensory neurons of Aplysia. Neuron 10:921-929.

Jan LY, Jan YN (1976a) Properties of the larval neuromuscular junction of Drosophila melanogaster. J Physiol (Lond) 262:215-236.

Jan LY, Jan YN (1976b) L-glutamate as an excitatory transmitter at the Drosophila larval neuromuscular junction. J Physiol (Lond) 262:189-214.

Jia X, Gorczyca M, Budnik V (1993) Ultrastructure of neuromuscular junctions in Drosophila: comparison of wild type and mutants with increased excitability. J Neurobiol 24:1025-1044.

Katz LC, Shatz CJ (1996) Synaptic activity and the construction of cortical circuits. Science 274:1133-1138.

Keshishian H, Chiba A, Chang TN, Halfon MS, Harkins EW, Jarecki J, Wang L, Anderson MD, Cash S, Halpern ME, Johansen J (1993) Cellular mechanisms governing synaptic development in Drosophila melanogaster. J Neurobiol 24:757-787.

Keshishian H, Broadie KS, Chiba A, Bate M (1996) The Drosophila neuromuscular junction: a model system for studying synaptic development and function. Annu Rev Neurosci 19:545-575.

Kim YT, Wu C-F (1996) Reduced growth cone motility in cultured neurons from Drosophila learning and memory mutants with a defective cAMP cascade. J Neurosci 16:5593-5602.

Kurdyak P, Atwood HL, Stewart BA, Wu C-F (1994) Differential physiology and morphology of motor axons to ventral longitudinal muscles in larval Drosophila. J Comp Neurol 350:463-472.

Kurjak M, Fritsch R, Saur D, Schusdziarra V, Allescher HD (1999) NO 
releases bombesin-like immunoreactivity from enteric synaptosomes by cross-activation of protein kinase A. J Am Physiol 276:G1521-1530.

Kuromi H, Kidokoro Y (1998) Two distinct pools of synaptic vesicles in single presynaptic boutons in a temperature-sensitive Drosophila mutant, shibre. Neuron 20:917-925.

Levin LR, Han P-L, Hwang PM, Feinstein PG, Davis RL, Read RR (1992) The Drosophila learning and memory gene rutabaga encodes a calcium/calmodulin-responsive adenylyl cyclase. Cell 68:479-489.

Li W, Tully T, Kalderon D (1995) Effects of a conditional Drosophila PKA mutant on olfactory learning and memory. Learn Mem 2:320-333.

Lindsley DL, Zimm GG (1992) The genome of Drosophila melanogaster. San Diego: Academic.

Livingstone MS, Sziber PP, Quinn WG (1984) Loss of calcium/calmodulin responsiveness in adenylate cyclase of rutabaga, a Drosophila learning mutant. Cell 37:205-215.

Meinertzhagen IA, Emsley JG, Sun XJ (1998) Developmental anatomy of the Drosophila brain: neuroanatomy is gene expression. J Comp Neurol 402:1-9.

Nguyen PV, Kandel ER (1996) A macromolecular synthesis-dependent late phase of long-term potentiation requiring cAMP in the medial perforant pathway of rat hippocampal slices. J Neurosci 16:3189-3198.

Pieribone,VA, Shupliakov O, Brodin L, Hilfiker-Rothenfluh S, Czernik AJ, Greengard P (1995) Distinct pools of synaptic vesicles in neurotransmitter release. Nature 375:493-497.

Renger JJ, Stewart BA, Atwood HL, Wu C-F (1995) Focal loose-patch recordings of identifiable boutons in the learning mutants dunce and rutabaga show altered calcium dependence and synaptic plasticity. Neurosci Abstr 21:169.

Renger JJ, Ueda A, Wu C-F (1998) Electrophysiological and optical measurements reveal a gradient of synaptic efficacy within motor terminal arbors of the larval neuromuscular junction in wild-type and cAMP-cascade mutants of Drosophila. Neurosci Abstr 24:1777.

Renger JJ, Yao W-D, Sokolowski MB, Wu C-F (1999) Neuronal polymorphism among natural alleles of a cGMP-dependent kinase gene, foraging, in Drosophila. J Neurosci 19:RC28.

Schuster CM, Davis GW, Fetter RD, Goodman CS (1996) Genetic dissection of structural and functional components of synaptic plasticity. II. Fasciclin II controls presynaptic structural plasticity. Neuron 17:655-667.

Stevens CF, Tsujimoto T (1995) Estimates for the pool size of releasable quanta at a single central synapse and for the time required to refill the pool. Proc Natl Acad Sci USA 92:846-849.

Stewart BA, Atwood HL, Renger JJ, Wang J, Wu C-F (1994) Im- proved stability of Drosophila larval neuromuscular preparations in haemolymph-like physiological solutions. J Comp Physiol [A] 175:179-191.

Stewart BA, Schuster CM, Goodman CS, Atwood HL (1996) Homeostasis of synaptic transmission in Drosophila with genetically altered nerve terminal morphology. J Neurosci 16:3877-3886.

Stühmer W, Roberts WM, Almers W (1983) The loose patch clamp. In: Single-channel recording (Sakmann B, Neher E, eds), pp 123-132. New York: Plenum.

Südhof TC (1995) The synaptic vesicle cycle: a cascade of proteinprotein interactions. Nature 375:645-653.

Torphy TJ (1994) Beta-adrenoceptors, cAMP and airway smooth muscle relaxation: challenges to the dogma. Trends Pharmacol Sci 15:370-374.

Tully T, Quinn WG (1985) Classical conditioning and retention in normal and mutant Drosophila melanogaster. J Comp Physiol [A] 157:263-277.

Wang J, Renger JJ, Griffith LC, Greenspan RJ, Wu C-F (1994) Concomitant alteration of physiological and developmental plasticity in Drosophila CaM kinase II-inhibited synapses. Neuron 13:1373-1384.

Weible ER, Kistler GS, Scherle WF (1969) Practical stereological methods for morphometric cytology. J Cell Biol 30:23-33.

Yao W-D, Rusch J, Poo M-M, Wu C-F (2000) Spontaneous acetylcholine secretion from developing growth cones of Drosophila central neurons in culture: effects of cAMP-pathway mutations. J Neurosci 20:2626-2637.

Yin JCP, Wallach JS, Del Vecchio M, Wilder EL, Zhou H, Quinn WG, Tully T (1994) Induction of a dominant negative CREB transgene specifically blocks long-term memory in Drosophila. Cell 79:49-58.

Zhao M-L, Wu C-F (1997) Alterations in frequency coding and activity dependence of excitability in cultured neurons of Drosophila memory mutants. J Neurosci 17:2187-2199.

Zhong Y, Wu C-F (1991) Altered synaptic plasticity in Drosophila memory mutants with a defective cAMP cascade. Science 251:198-201.

Zhong Y, Wu C-F (1993) Differential modulation of potassium currents by cAMP and its long-term and short-term effects: dunce and rutabaga mutants of Drosophila. J Neurogenet 9:15-27.

Zhong Y, Budnik V, Wu C-F (1992) Synaptic plasticity in Drosophila memory and hyperexcitability mutants: role of cAMP cascade. J Neurosci 12:644-651.

Zucker RS (1989) Short-term synaptic plasticity. Annu Rev Neurosci 12:13-31. 